\title{
Compact Printed Arrays with Embedded Coupling Mitigation for Energy-Efficient Wireless Sensor Networking
}

\author{
Constantine G. Kakoyiannis and Philip Constantinou \\ Mobile Radio Communications Laboratory, National Technical University of Athens, 9 Heroon Polytechniou Sreet, \\ Zographos Polytechnic Campus, 15773 Athens, Greece \\ Correspondence should be addressed to Constantine G. Kakoyiannis, kkak@mobile.ntua.gr
}

Received 1 December 2009; Accepted 1 April 2010

Academic Editor: Hoi Shun Lui

Copyright ( 2010 C. G. Kakoyiannis and P. Constantinou. This is an open access article distributed under the Creative Commons Attribution License, which permits unrestricted use, distribution, and reproduction in any medium, provided the original work is properly cited.

\begin{abstract}
Wireless sensors emerged as narrowband, resource-constrained devices to provide monitoring services over a wide life span. Future applications of sensor networks are multimedia-driven and include sensor mobility. Thus, sensors must combine small size, large bandwidth, and diversity capabilities. Compact arrays, offering transmit/receive diversity, suffer from strong mutual coupling (MC), which causes lower antenna efficiency, loss of bandwidth, and signal correlation. An efficient technique to reduce coupling in compact arrays is described herein: a defect was inserted in the ground plane (GNDP) area between each pair of elements. The defect disturbed the GNDP currents and offered multidecibel coupling suppression, bandwidth recovery, and reduction of in-band correlation. Minimal pattern distortion was estimated. Computational results were supported by measurements. The bandwidth of unloaded arrays degraded gracefully from $38 \%$ to $28 \%$ with decreasing interelement distance $(0.25 \lambda$ to $0.10 \lambda)$. Defect-loaded arrays exhibited active impedance bandwidths 37-45\%, respectively. Measured coupling was reduced by $15-20 \mathrm{~dB}$.
\end{abstract}

\section{Introduction}

It has been fifteen years since wireless sensor network (WSN) pioneers first envisioned the cubic-millimeter "Smart Dust" sensor node (or simply "mote") [1,2]. Yet, studies on the very thing that makes WSN's wireless are scarce; the work in [3] is but an exception to this norm. However, there are certain facts concerning the development of WSNs that draw attention to the antenna system.

1.1. Antenna Design in the Context of Sensor Networking. Sensor devices are severely constrained in terms of battery, memory, processing capability, transmitted power, and achievable data rate. They were to communicate over short distances, but the multihop communication paradigm proved inefficient; data packets are delivered more efficiently with two or three longer hops [4]. Achievable rate is linked to power consumption. Motes operate with nonreplaceable batteries, but, still, from a system deployment perspective, lifetimes measured in years are required for most building, industrial, and forestry applications [2]. Energy consumption is a fundamental issue associated with network lifetime and connectivity. Additionally, the energy expenditure of the analog RF part overwhelms that of other sensor node operations, which actually makes RF the bottleneck for lifetime improvement [2]. Because of this, the efficiency of the antenna system is directly connected to the energy efficiency of the overall node decibel-for-decibel.

Future applications of sensor networks also include sensor mobility $[5,6]$. Mobility combined with the requirement for robust transmissions over longer hops calls for a study of the feasibility of compact array implementation on motes with realistic dimensions: taking indoor WSN deployments as an example, multipath propagation effects cause reliability problems, since time-varying narrowband fading of tens of decibels is commonly observed [2]. Arrays offer the possibility of employing low-complexity transmit/receive diversity schemes, which enable sensor mobility. Hence, the de facto specifications of small size and low-cost manufacturing for WSN-targeted antennas are incremented by the requirements for high total efficiency and array formation. Mutual coupling threatens to make the two latter specifications mutually exclusive. 
Antennas are the most neglected circuits-and-systems aspect of sensor nodes and networks. This has brought about difficulties in integrating antennas into microsensors, and it was suggested that either frequencies beyond $10 \mathrm{GHz}$ or inefficient chip antennas are used [2]. Despite the lack of studies on WSN-targeted antennas, wireless sensors are cross-layer entities and thus optimization of algorithmic and networking aspects has taken place under the assumption that the antenna system possesses certain properties. For example, energy-efficient medium access solutions have been theorized under the assumption of antenna directivity, either switched-beam or beamformed [7, 8], implemented as uniform linear or circular arrays. The same holds for routing algorithms [9, 10]. Information-theoretic studies have utilized elements of antenna systems to extend the connectivity and lifetime of WSNs [11]. Ultra-wideband implementations of WSNs for localization at millimeterwave frequencies have been proposed again under the assumption of antenna directivity [12]. Still, little has been done to address the challenges and complexities of antenna implementation for WSNs, and especially arrays; two recent efforts are described in $[13,14]$. By WSN standards, both studies resulted in bulky prototypes, attesting the fact that a lot of ground needs to be covered in this area.

To the best of the authors' knowledge, the work by Abbosh and Thiel [15] is the only existing study on the effect of mutual coupling on wireless sensors. The authors developed a theoretical model that relates the energy expenditure per transmitted bit to the presence of mutual coupling between the elements of uniform circular arrays in $2 \times 2$ and $4 \times 4$ MIMO systems. Mutual coupling was incorporated in circuit terms into the channel matrix and lead to a modified MIMO channel matrix. Line-of-sight (LOS) propagation conditions were assumed, and the study opted for capacity maximization. To this end, full knowledge of channel state information (CSI) was assumed at the transmitter, and waterfilling adjusted properly the power transmitted from each antenna element. The authors concluded that mutual coupling is beneficial for such a configuration, since it leads to a decorrelated modified channel matrix and decreases the required energy per bit. The work presented herein diverges significantly from [15] in terms of fundamental assumptions.

(i) The complexity introduced by the multiple receive and transmit RF chains is unrealistic, at least by current CMOS standards and implementation costs.

(ii) CSI availability at the transmitter introduces overhead (feedback) and severe computational complexity, because of the need for real-time matrix inversions [16]. Full CSI knowledge is a common assumption made in algorithmic MIMO studies, but in every other aspect, it is very difficult to be hardcoded at the FPGA or DSP level.

(iii) In both indoor and outdoor WSN implementations, LOS propagation conditions are not given; rather, NLOS conditions should be expected. Consider, for example, deployment in office floors and in forests. Thus, energy efficiency will be obtained through link robustness (diversity gain) and not through capacity maximization.

(iv) Spatial multiplexing techniques work well only above a threshold SNR value at the receiver, for example, $\mathrm{SNR}_{\min }=20 \mathrm{~dB}$. Such high $\mathrm{SNR}$ values are not guaranteed in severe NLOS conditions. Below the given threshold, only diversity gain can be sought.

1.2. Mutual Coupling Reduction in Compact Arrays. In what follows, antenna spacing refers to the distance of the feed points, not edge-to-edge. Important dimensions are expressed in terms of free-space wavelength $\lambda$.

Yang and Rahmat-Samii [17] presented an early study of mutual coupling suppression based on electromagnetic band-gap (EBG) structures. Two patch antennas were printed on a thick substrate and spaced at $d=0.75 \lambda$. A $14 \times 4$ matrix of mushroom-like patches was printed between the antennas. The technique produced a coupling reduction of $8.8 \mathrm{~dB}$ and occupied an area $1.07 \lambda \times 0.30 \lambda$.

Dossche et al. [18] suggested the use of a combination of matching and decoupling network to suppress the correlation coefficient. The achieved resonance was very deep but also narrowband (1.6\%), since the circuit was based on transmission line sections. The required PCB area measured $1.43 \lambda \times 1.34 \lambda$.

A different type of ground defect was studied and presented very well in [19]. The authors built two planar inverted-F antennas (IFAs) on a square PCB and spaced them at $d=0.12 \lambda$. Two arrays of slits where then etched away on opposite sides on the GND plane, between the two antennas. The disturbance caused to the ground currents offered a coupling reduction in the range $8-16 \mathrm{~dB}$ for various antenna scenarios. The slits occupied a PCB area $0.33 \lambda \times 0.07 \lambda$.

Kokkinos et al. [20] built two planar IFAs on a long $\mathrm{PCB}$ and spaced them at $d=0.62 \lambda$. Two slits where then etched away on opposite sides on the GND plane, between the two antennas. The authors optimized the dimensions and spacing of the slits, to make them resonate like a magnetic loop. In the band of interest, coupling reduction ranged between 10 and $40 \mathrm{~dB}$. The slits occupied an area $0.25 \lambda \times$ $0.14 \lambda$. A single slit was also applied in [21] to decouple two planar IFAs. The difference was that the slit was not etched between the elements, but further down the GND plane. The size of the slit was $0.18 \lambda \times 0.12 \lambda$.

The authors in [22] also studied the application of electromagnetic band-gap (EBG) structures. Two horizontal dipole antennas were mounted on a substrate and spaced at $d=0.17 \lambda$. A $9 \times 3$ matrix of mushroom-like patches was printed between the antennas. The technique produced an 8 - $\mathrm{dB}$ coupling reduction and occupied an area $0.56 \lambda \times 0.11 \lambda$.

EBG was also the method of choice for Michailidis et al. [23]. Two patch antennas were printed on a substrate and spaced at $d=0.67 \lambda$. A $9 \times 3$ matrix of mushroom-like patches was printed between the antennas. The periodic structure occupied an area $0.60 \lambda \times 0.20 \lambda$.

1.3. Scope and Outline of the Paper. This work focuses on the straightforward and low-cost implementation of 
compact printed arrays suitable for WSN-targeted diversity applications. An effective MC suppression technique was developed, which is compatible with PCB printing and practically introduces no extra cost of implementation. Mutual coupling hinders diversity, since it degrades energy efficiency in transmit mode (through a decrease in antenna efficiency), and decreases the total received power in receive mode [24]. Moreover, it detunes the elements and leads to loss of bandwidth.

The paper is organized as follows. Section 2 starts with a brief description of the sinusoidal monopole, the compact element used as a building block to design compact arrays for motes. Measured data harvested from eight prototype antennas are given. The study of a compact two-element array begins next. The modeling procedure is described, together with the numerical and measurement results that mark the extent of coupling and signal correlation. The effect of element proximity in terms of detuning and VSWR bandwidth, in the presence of strong mutual coupling, was studied. To test for benefit in diversity systems, the correlation coefficient as a function of interelement distance was calculated.

In case of severe correlation, a simple yet efficient technique to reduce the coupling is proposed: the return currents on the GND plane are disturbed by a nonperiodic photonic bandgap (PBG) structure known as the defected ground structure (DGS). This effort is a straightforward and cost-effective step towards the fabrication of square-inch diversity antenna arrays for sensor networks. The technique is presented in detail in Section 3 and it is backed by measured data harvested from six prototype arrays.

Section 4 describes the improvement in both radiation and total efficiency that is achieved by embedding just a single DGS cell in the compact array. Improvement of both types of efficiency implies that this method offers mutual coupling reduction and bandwidth recovery. The minimal distortion of the far-field pattern is also attested through accurate computational models. Finally, Section 5 concludes the work.

\section{System Model and Electrical Performance of a Compact Printed Array}

Future applications of sensor networks are multimedia driven and include sensor mobility. In a time-varying NLOS environment such as the one encountered in mobile sensor networks, antenna arrays offer the ability to employ transmit-receive diversity schemes, and thus they can function as true enablers of sensor mobility. Before delving into the details of compact arrays in Section 2.2, the following section presents briefly the array building block, along with pertinent measurement results.

2.1. Sinusoidal Antennas: Description and Measured Data. Antennas are immune to miniaturization, because the physical laws that determine their behavior produce selfconflicting fundamental attributes: the efficiency-bandwidth product (EBWP) increases with the volume occupied by

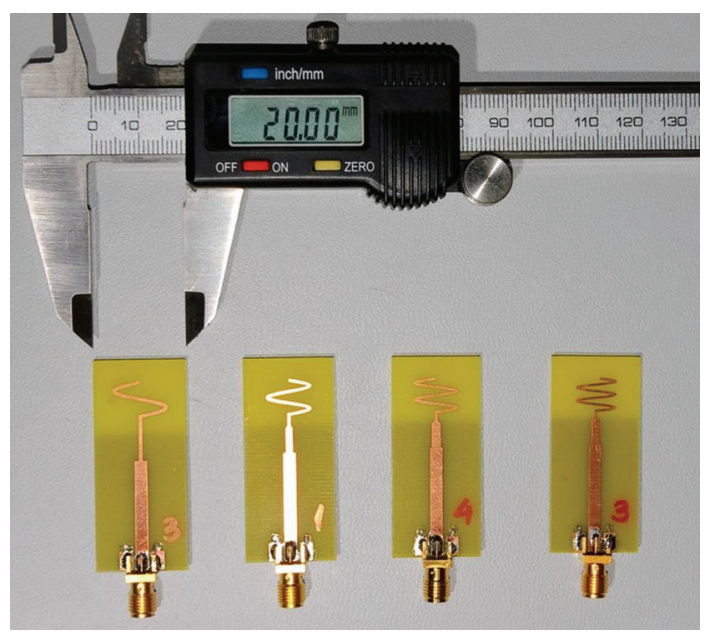

FIgURE 1: Evolution of the sinusoidal antenna. From left to right, the line-up shows iterations $i=2,3,4,5$. All monopoles are mirrored against the nominal GNDP with a size of $20 \mathrm{~mm} \times 30 \mathrm{~mm}$. Notice that as iteration grows, element size shrinks; it was found to converge at $i=6$.

the antenna. The theoretical foundations of small antennas predict that good performance is obtained when most of the allocated space participates in radiation. Thus, printed antennas, which are inherently 2D structures, seem a priori handicapped as small efficient radiators for WSN nodes. Nevertheless, the work in [25-28] showed that this is not so. The printed sinusoidal monopole proposed in [25] is a way to reengineer the meander-line antenna [29]. Shaping the meander like a smooth sinusoidal curve produced small antennas exhibiting a second frequency-adjacent resonance; this feature improved the operational VSWR bandwidth $\left(\mathrm{BW}_{\mathrm{V}}\right)$ greatly.

Figure 1 depicts the antennas under study; they operate at $2.5 \mathrm{GHz}$ where $\lambda=120 \mathrm{~mm}$. The two-layer PCB is made of low-cost FR4 material, whereas its size represents the whole size of the sensor node. The substrate properties that were assumed at $2.5 \mathrm{GHz}$ were $\varepsilon_{r}=4.6, \tan \delta=0.0170$, and $H=1.60 \mathrm{~mm}$. On the Top layer, the printed antenna and the microstrip line that excites it were etched. On the Bottom layer, a continuous copper cladding serves as the GNDP of both the antenna and the microstrip (shown in Figure 1 as a shadow on the back). The GNDP was removed below the antenna. It was assumed that all RF and baseband ICs and discrete components would be soldered on the Bottom layer in an actual WSN node implementation. The antenna element comprises an initial straight segment and an integer number of half-periods of a sinusoidal curve. The number of half-periods, $i$, is the iteration of the antenna. Figure 1 actually shows the sinusoidal evolution from the second to the fifth iteration.

Computed impedance bandwidth and radiation efficiency results were reported in detail in [25-28]. Measured data collected from the prototype antennas depicted in Figure 1 are reported here. The measurement setup is described, and the discrepancies between computed and measured results are accounted. The measurements took 
International Journal of Antennas and Propagation

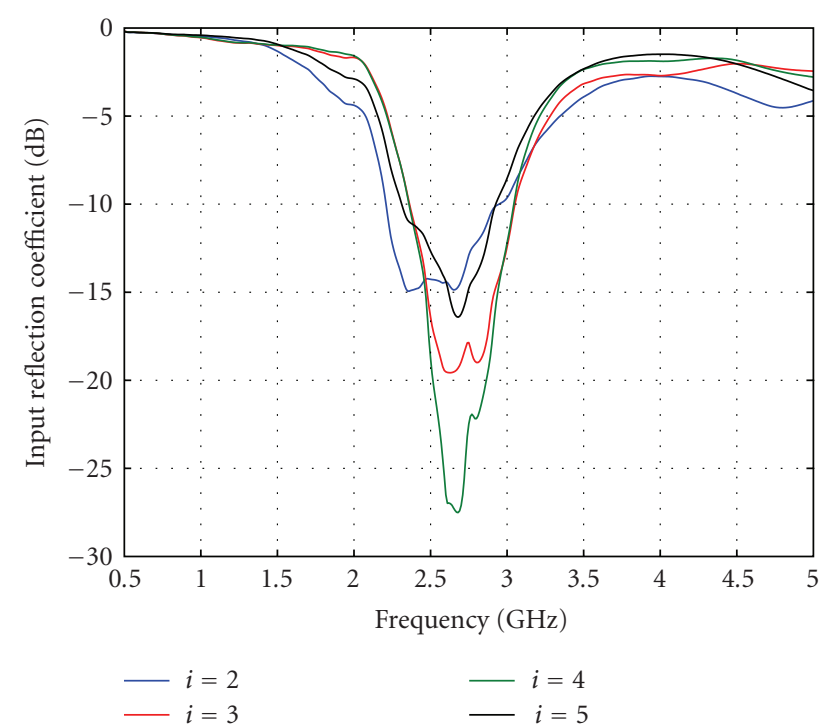

Figure 2: Measured reflection coefficient and impedance bandwidth of the four sinusoidal iterations shown in Figure 1, which featured the common nominal GNDP size (overall PCB size differs).

place at the Laboratory, and not inside an anechoic facility. However, measures were taken to minimize the effect of the surroundings; the antennas were mounted on small Styrofoam tables that separated them from the bench, while absorbing material was used to cover surrounding objects and create an isolated environment. An Agilent PNA-L N5230A vector network analyzer (VNA) recorded the complex scattering parameters of each antenna under test (AUT). The VNA was calibrated in the $0.5-5 \mathrm{GHz}$ frequency range. Low-loss coaxial cables were used during VNA calibration and measurement.

The measured data shown in Figure 2 indicated achievable bandwidth $\mathrm{BW}_{\mathrm{V} \text {,meas }}=0.61-0.73 \mathrm{GHz}$, or in fractional terms $\mathrm{FBW}_{\mathrm{V} \text {,meas }}=23.5-28.5 \%$. The average centre frequency of the four resonances was $2.65 \mathrm{GHz}$. By comparison of the measured data to the simulation results in [25-28], the following differences are noted: (a) the upper resonance at $3.2 \mathrm{GHz}$ did not appear in the measurements, and (b) the lower resonance shifted from $2.5 \mathrm{GHz}$ to the range 2.62.7 GHz. Still, the measured sinusoidal antennas achieved adequate VSWR bandwidths despite their small size. The achievable bandwidths are much greater than needed for video transmission, for example, in surveillance sensor networks. In such networks, MPEG-coded video traffic demands only a few megahertz per channel. Such great bandwidths could even accommodate uncoded video transmissions. The designer is offered the choice to trade some of the available $\mathrm{BW}_{\mathrm{V}}$ for further antenna miniaturization. The $\mathrm{BW}_{\mathrm{V}}$ is defined at VSWR $=1.92 \simeq 2.0$, that is, for a reflection coefficient magnitude $\left|S_{11}\right|=-10 \mathrm{~dB}$.

The disagreement between simulated and measured results was caused by the different GNDP sizes (the results in [25-28] correspond to optimized GNDP dimensions) and the substrate material. The design was carried out under the assumption that fabrication would take place on a 1.60mm thick FR-4 material that would exhibit $\varepsilon_{r}=4.6$ and
TABLE 1: Measured bandwidth before and after the optimization of GNDP dimensions.

\begin{tabular}{|c|c|c|c|c|c|c|}
\hline$i$ & $\begin{array}{c}\text { Nominal } \\
\text { GNDP } \\
\text { BW }_{\mathbf{V}} \\
(\mathrm{GHz}) \\
\end{array}$ & $\begin{array}{c}\text { Nominal } \\
\text { GNDP } \\
\text { FBW }_{\mathrm{V}} \\
(\%) \\
\end{array}$ & $\begin{array}{c}\text { Optimal } \\
\text { GNDP } \\
\text { BW }_{\mathbf{V}} \\
(\mathrm{GHz}) \\
\end{array}$ & $\begin{array}{c}\text { Optimal } \\
\text { GNDP } \\
\text { FBW }_{\mathrm{V}} \\
(\%) \\
\end{array}$ & $\begin{array}{c}\text { BW }_{\mathbf{V}} \\
\text { improve- } \\
\text { ment } \\
(\%)\end{array}$ & $\begin{array}{c}\mathrm{FBW}_{\mathrm{V}} \\
\text { improve- } \\
\text { ment } \\
(\%) \\
\end{array}$ \\
\hline 2 & 0.730 & 28.3 & 1.032 & 38.0 & 41.4 & 34.3 \\
\hline 3 & 0.688 & 25.4 & 0.916 & 33.2 & 33.1 & 30.7 \\
\hline 4 & 0.685 & 25.4 & 0.896 & 32.8 & 30.8 & 29.1 \\
\hline 5 & 0.613 & 23.4 & 0.799 & 29.4 & 30.3 & 25.6 \\
\hline
\end{tabular}

$\tan \delta=0.0170$ at $2.5 \mathrm{GHz}$. As it turned out, there is no FR-4 material exhibiting such a high dielectric constant in the gigahertz range. The PCB was fabricated on $1.47-\mathrm{mm}$ thick Isola IS400 material, specified to exhibit $\varepsilon_{r}=5.0$ and $\tan \delta=0.0140$ at $1 \mathrm{MHz}$. After building, measuring and back-simulating a simple resonant microstrip circuit on this substrate, the dielectric constant at $2.5 \mathrm{GHz}$ was estimated at $\varepsilon_{r, 2.5 \mathrm{GHz}}=4.28$. The problem with using a lower permittivity substrate is obvious: the antennas became inductive and electrically larger (i.e., resonated higher), due to lesser dielectric loading. The deviation is small, because the change in dielectric constant was partially alleviated by the reduction in substrate height.

The lack of sufficient information from FR-4 vendors on the dispersive behavior of materials is another source of discrepancy. Accurate modeling and simulation demand accurate first- or second-order Debye models of dielectric material dispersiveness, instead of single-frequency data. Low-cost FR4 substrates exhibit spatially nonuniform loss and dielectric constant, causing inconsistent $\mathrm{E} / \mathrm{M}$ modeling. Nevertheless, this inconsistency is part of the trade-off of pragmatic antenna design for low-cost portable devices.

It is well known that the performance of a small-deviceintegrated antenna is not only a function of the current distribution on the element itself but also a function of the induced current distribution on the finite GNDP $[25,29-$ 31]. In fact, the GNDP becomes as much the antenna as the sinusoidal element itself. The effect of the dimensions of the respective GNDPs was studied using the methodology described in [29-31]. The elements and their feed components were left unchanged during this procedure. The GNDP adaptation study sought to optimize the aforementioned trade-off between total efficiency, fractional bandwidth, and electrical size of the joint radiator; the three parameters were weighted equally.

The four sinusoidal monopoles shown in Figure 1 were rebuilt on PCBs having the GNDP dimensions listed in [25-28]. The result was the four new prototypes shown in Figure 3. The measured data depicted in Figure 4 attest that the GNDP adaptation worked as intended: all four monopoles achieved greater bandwidths, while shallower resonances were also recorded (higher values of $\left|\Gamma_{\text {in }}(j \omega)\right|$ ). Thus, GNDP adaptation can produce smaller overall radiators with larger operating bandwidths by entering the unmatched and nonresonant region. The extent of GNDPinduced improvement is tabulated in Table 1. 


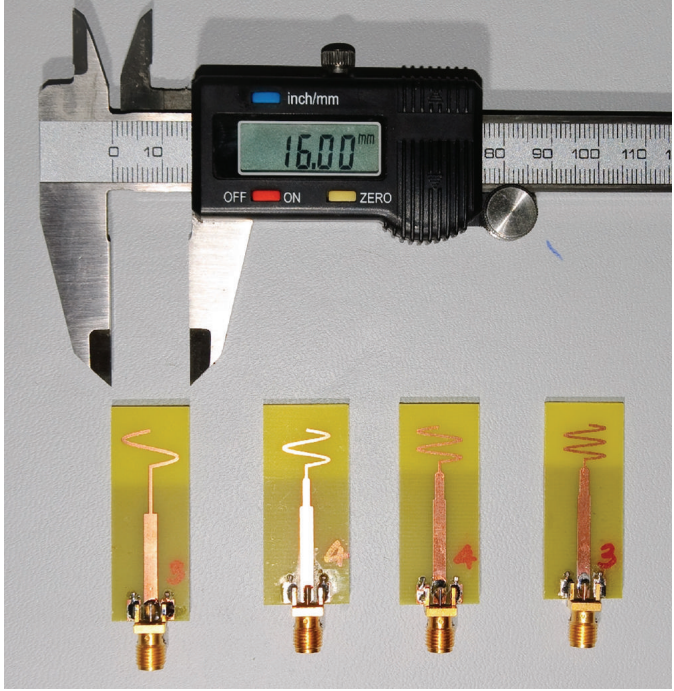

Figure 3: From left to right, the line-up shows iterations $i=$ $2,3,4,5$. The monopoles are mirrored against the respective optimal GNDP sizes [25-28]. The antennas were printed again on Isola IS400, so the problem with the lower permittivity persists: these monopoles were also inductive and slightly electrically larger.

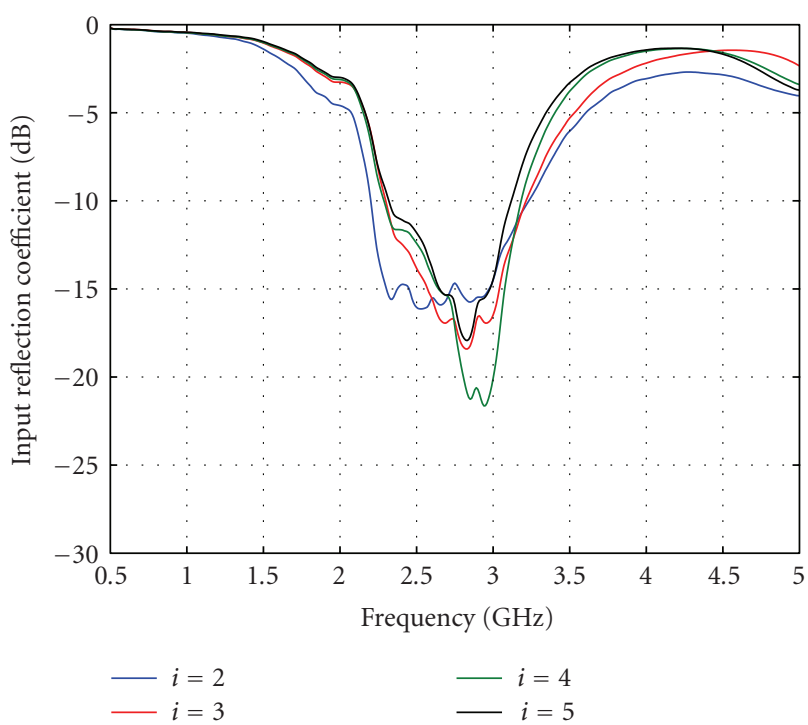

FIGURE 4: Measured reflection coefficient and impedance bandwidth of the four sinusoidal iterations shown in Figure 3. The inductive behavior was attested by Smith charts of $S_{11}(j \omega)$ during measurements.

2.2. Modeling the Compact Array. The following sections describe the design and performance of a two-element printed array built with sinusoidal elements and having variable interelement distance (IED), denoted by $d$ [26].

Two third-iteration sinusoidal monopoles $(i=3)$ were placed on a common PCB carrier; so their feed lines shared the same GNDP and the same substrate. Figure 5 depicts three such arrays that were fabricated in this context. The third-order sinusoidal element measures $10 \mathrm{~mm} \times 10 \mathrm{~mm}$. The optimal GNDP dimensions were calculated at $26 \mathrm{~mm} \times$ $18 \mathrm{~mm}$. The width of the sinusoidal copper trace equals

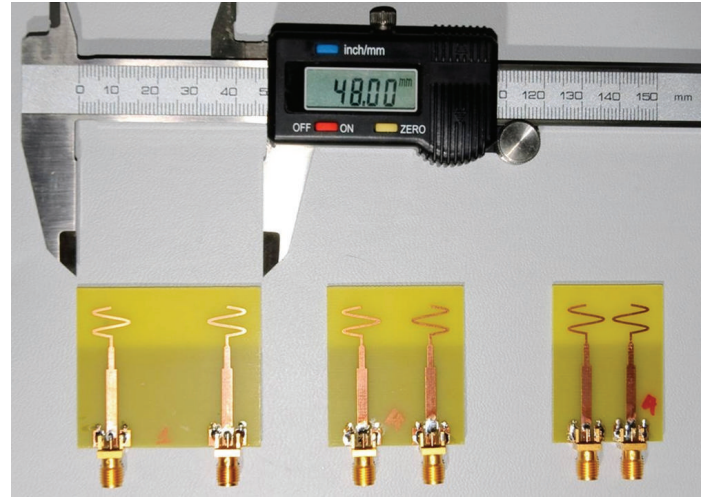

FIGURE 5: The top layer of three fabricated compact arrays. From left to right, the interelement distance is $30 \mathrm{~mm}(0.25 \lambda), 18 \mathrm{~mm}(0.15 \lambda)$, and $12 \mathrm{~mm}(0.10 \lambda)$ respectively.

$0.65 \mathrm{~mm}$. The last segment of the $2.7-\mathrm{mm}$ wide microstrip feeding line measures $6.0 \mathrm{~mm} \times 1.5 \mathrm{~mm}$ and acts as a series tuning inductance. Sinusoidal monopoles coupled to their ground planes provide readily available sensor node configurations at the square-inch and cubic-centimeter scales (in terms of occupied area and volume, resp.) [25-28].

The distance from the feed points to the respective sides of the PCB was $9 \mathrm{~mm}$; that is, it was set at half the optimum GNDP width [25-28]. The interelement distance is a design variable. To facilitate simulation, ports placed on equivalent square end-launch SMA connectors excited the feed lines. Surface current distributions (SCDs) were used in earlier studies to visualize the strong coupling between two antennas separated by $d=0.15 \lambda[26,27]$. The following sections quantify the severity of this coupling.

2.3. Array Parametric Study and Numerical Results. Interelement distance varied from $0.25 \lambda(=30 \mathrm{~mm})$ down to $0.10 \lambda$ $(=12 \mathrm{~mm})$. Under rich scattering conditions, diversity gain starts to deteriorate when IED drops below $0.2 \lambda$ [24], hence the choice of the range of $d$. Figure 6 depicts the broadband input matching. This is actually the active input impedance of the array, since it corresponds to one port being excited while the other is match terminated at $50 \Omega$ [32]. By comparison to the single-element simulation results in [2528 ], it is obvious that the upper adjacent resonance has vanished, while the first resonances shifted lower. This was due to the wider GNDP, which does not benefit sinusoidal antennas. However, large bandwidths were obtained in the $2-3 \mathrm{GHz}$ range; they ranged from $0.5 \mathrm{GHz}$ to $0.7 \mathrm{GHz}$. The respective fractional bandwidths ranged from $20 \%$ to $28 \%$. The resonances were in the range $2.32<f_{\text {res }}<2.48 \mathrm{GHz}$, whereas the center frequencies were in the range $2.39<f_{c}<$ $2.51 \mathrm{GHz}$.

Moreover, the results in Figure 7 show the changes in broadband mutual coupling given by the scattering parameter $S_{21}(j \omega)$. Worst-case coupling reached $S_{21}^{\max }=-4.5 \mathrm{~dB}$ for the smallest interelement distance. Such strong coupling makes the suitability of any antenna array questionable for diversity/MIMO systems. Only at $\lambda / 4$ spacing was the 


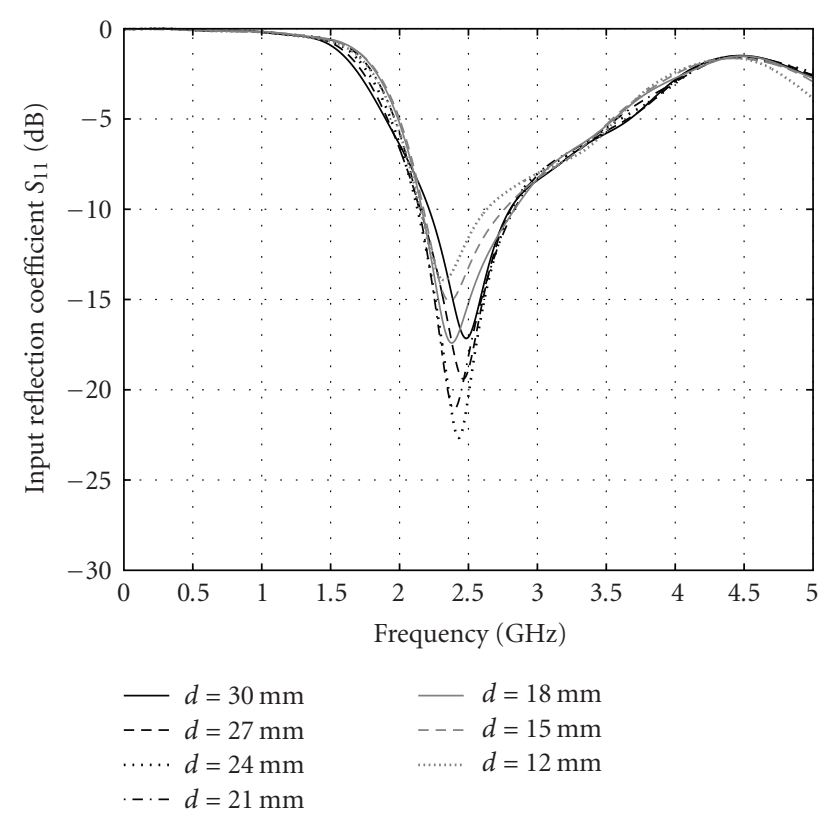

Figure 6: Parametric study of the broadband active input impedance for different values of interelement distance $(0.25 \lambda$ to $0.10 \lambda)$.

coupling reduced down to $-10 \mathrm{~dB}$. To test for diversity potential, signal correlation was expressed in terms of the complex scattering parameters of the 2-port array. For two antennas, the correlation between the envelopes of the signals, $\rho_{e}$, can be approximated by the square magnitude of the complex correlation coefficient $\rho_{c}[24,33,34]$ :

$$
\rho_{e} \simeq \frac{\left|S_{11}^{*} S_{12}+S_{21}^{*} S_{22}\right|^{2}}{\left(1-\left(\left|S_{11}\right|^{2}+\left|S_{21}\right|^{2}\right)\right)\left(1-\left(\left|S_{12}\right|^{2}+\left|S_{22}\right|^{2}\right)\right)} .
$$

Using $S$-parameters instead of far-field data for this estimation implicitly assumes that the propagation environment near the receiver is described by a uniform 3D angular power spectrum $[24,34]$. In the WSN case this holds approximately, because large angular spreads occur in WSNs (e.g., indoor and forestry deployments). In the event of a reciprocal and symmetrical array, (1) can be simplified as in:

$$
\rho_{e, \text { sym }} \simeq\left(\frac{2 \operatorname{Re}\left\{S_{11} S_{21}^{*}\right\}}{1-\left|S_{11}\right|^{2}-\left|S_{21}\right|^{2}}\right)^{2} .
$$

The estimation of $\rho_{e}$ via the $S$-parameters is convenient because it is fast and broadband. However, it must be used with caution, in light of the two possible sources of error described at the beginning of Section 3. The results in Figure 8 show great promise: the correlation coefficient was estimated below 0.35 even for a spacing $d_{\min }=12 \mathrm{~mm}=$ $0.10 \lambda$.

2.4. Array Measurement Results. The computational results were backed by measurements on three fabricated arrays. The 2-element arrays shown in Figure 5 were built on PCBs having a length $\operatorname{sub} L_{\text {array }}=26 \mathrm{~mm}$, as suggested in [26-29].

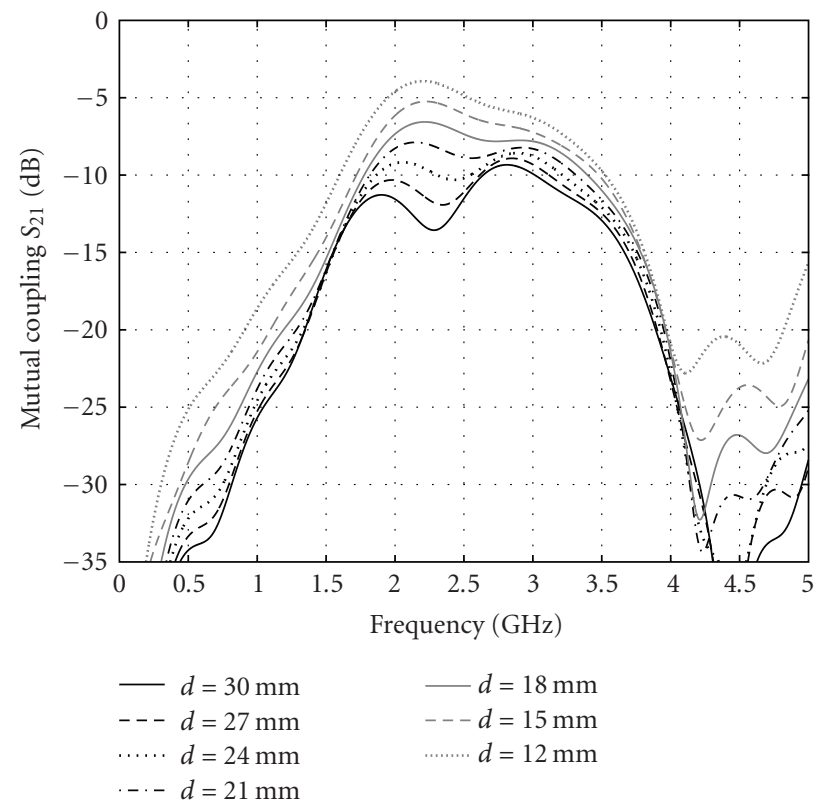

Figure 7: Parametric study of the broadband mutual coupling for different values of interelement distance $(0.25 \lambda$ to $0.10 \lambda)$.

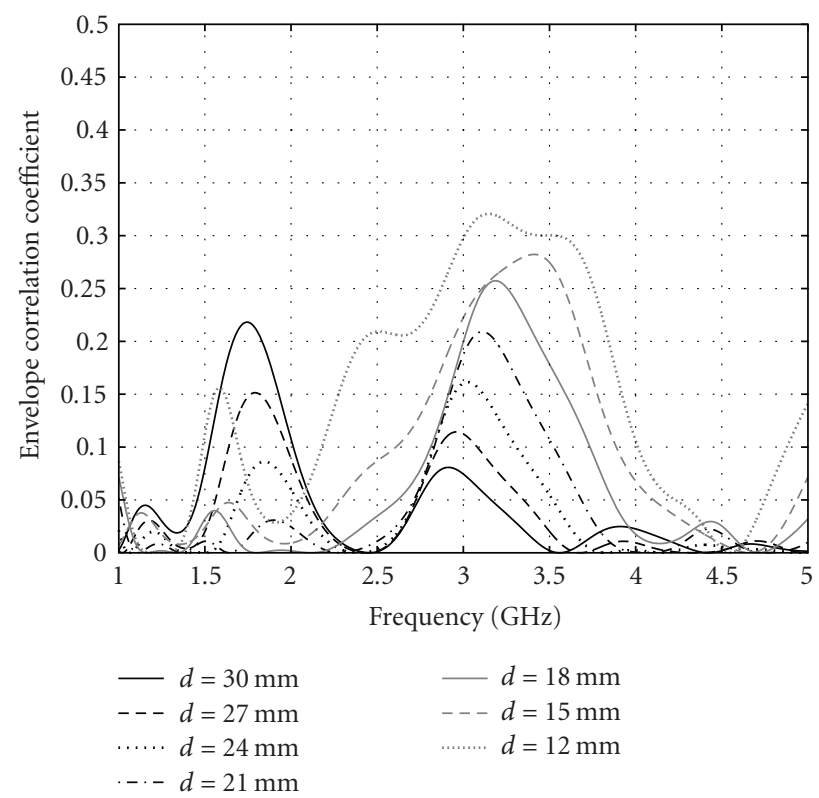

Figure 8: Parametric study of the broadband correlation coefficient for different values of interelement distance $(0.25 \lambda$ to $0.10 \lambda)$.

PCB width varied with interelement distance $d$ as in (3) (see Appendix A for a complete parametric nomenclature):

$$
\operatorname{sub} W_{\text {array }}=2 \frac{\operatorname{sub} W_{i=3}}{2}+d=\operatorname{sub} W_{i=3}+d,
$$

where $\operatorname{sub} W_{i=3}=18 \mathrm{~mm}$. The measurement setup was described in Section 2.1. The antennas were printed on Isola IS400; only this time the monopoles were embedded in a physically larger structure and were thus expected to respond as electrically smaller antennas. 


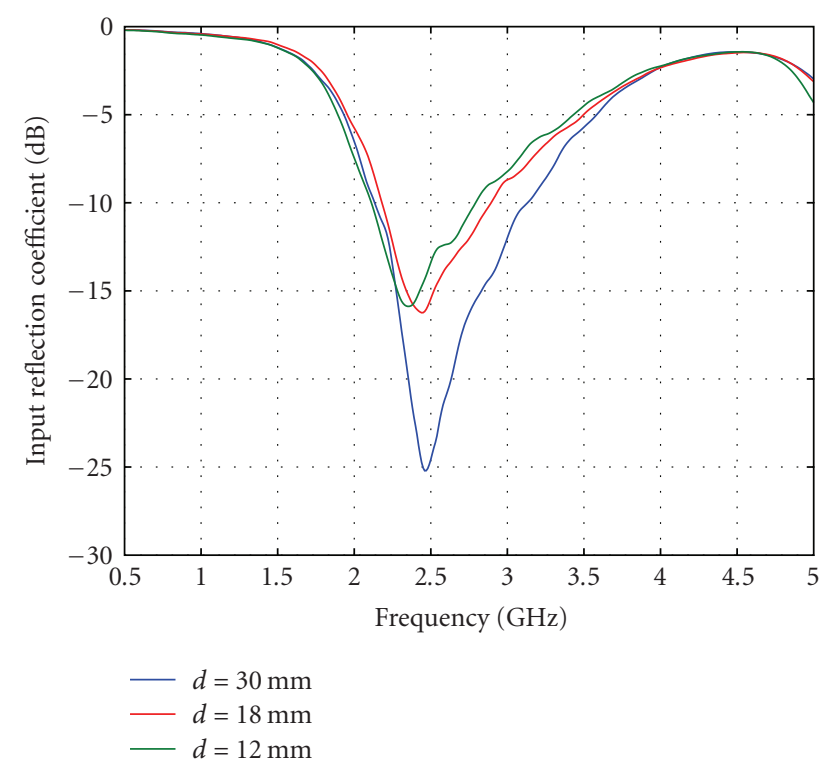

Figure 9: Measured active reflection coefficient and impedance bandwidth of the three 2-element arrays shown in Figure 5.

Indeed this response was recorded in Figure 9 and Table 2. All three resonances occurred below $2.5 \mathrm{GHz}$, contrary to what Figure 4 shows. At the largest IED, the active impedance bandwidth correlated well with the $\mathrm{BW}_{\mathrm{V}}$ of the nonarrayed element. Partial detuning occurred with decreasing IED. The measured mutual coupling in Figure 10 showed peak coupling that was stronger by $2 \mathrm{~dB}$ compared to numerically anticipated values.

\section{A Simple Technique That Reduces Mutual Coupling and Envelope Correlation}

There exist certain scenarios where it would be desirable or even mandatory to reduce coupling between the elements of the array. These cases include the following.

(i) The estimation of $\rho_{e}$ via the $S$-parameters might prove to be inaccurate due to low antenna efficiency. Although not mentioned in the original work [33], the expression derived by the authors is based on the power balance of impinging, coupled and radiated fields; therefore, it assumes total radiation efficiency equal to one [24].

(ii) The estimation of $\rho_{e}$ via the $S$-parameters might prove to be inaccurate due to the presence of scattering objects near the sensor node.

(iii) The feed points of the elements may need to be brought even closer than $\lambda / 10$.

Since both array elements are strongly coupled with their common GNDP, the significant current distribution on the GNDP is the dominant coupling factor, compared to feed separation distance and near-field coupling [24]. In this context, a simple way to achieve MC reduction is to use a defected ground structure (DGS). A single DGS

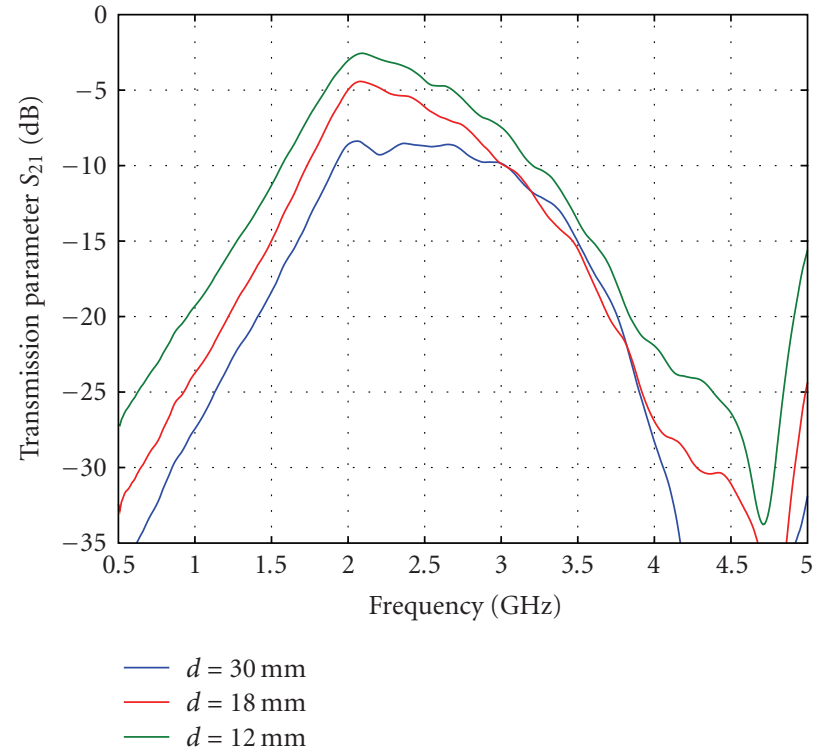

Figure 10: Measured mutual coupling between the elements of the arrays shown in Figure 5. Coupling was expressed in terms of $\left|S_{21}(j \omega)\right|$.

TABLE 2: Active input impedance bandwidths of the three measured arrays.

\begin{tabular}{lccc}
\hline$d(\mathrm{~mm})$ & Center frequency $(\mathrm{GHz})$ & $\mathrm{BW}_{\mathrm{V}}(\mathrm{GHz})$ & $\mathrm{FBW}_{\mathrm{V}}(\%)$ \\
\hline 30 & 2.632 & 0.995 & 37.8 \\
18 & 2.537 & 0.712 & 28.1 \\
12 & 2.452 & 0.680 & 27.7 \\
\hline
\end{tabular}

cell can be seen in Figures 11, 12, and 13. The proposed method is a decoupling technique aiming to reduce coupling, rather than completely cancel it; moreover, it does so in a broadband manner. Seen from a different perspective, the DGS decoupler attempts to maintain a given level of array efficiency and correlation (and thus diversity gain), despite the addition of more antennas into the WSN terminal.

3.1. Defected Ground Structures. Defected ground planes are an evolution of photonic bandgap (PBG) structures from the optical regime towards microwave frequencies [35]. Contrary to PBG structures, they are usually nonperiodic [36]. The DGS unit cell can be modeled near the resonance frequency as a parallel RLC circuit in series with and between two segments of transmission line. Park [37, 38] suggested a slightly more complex equivalent circuit by adding shunt resistances and capacitances at the two reference planes of the defect. That work also included a way to extract the values of the circuit elements from the ABCD-parameters of the twoport defect. The ABCD-parameters can be extracted from the $S$-parameters of the structure, which are obtained after simulation in E/M solvers or measurement of actual hardware. Karmakar et al. provided a quasistatic analysis of the most frequently occurred defect, that is, the dumbbell DGS [39]. Their analysis attempted to quantify the contribution from every part of the defect in the frequency response. Their 


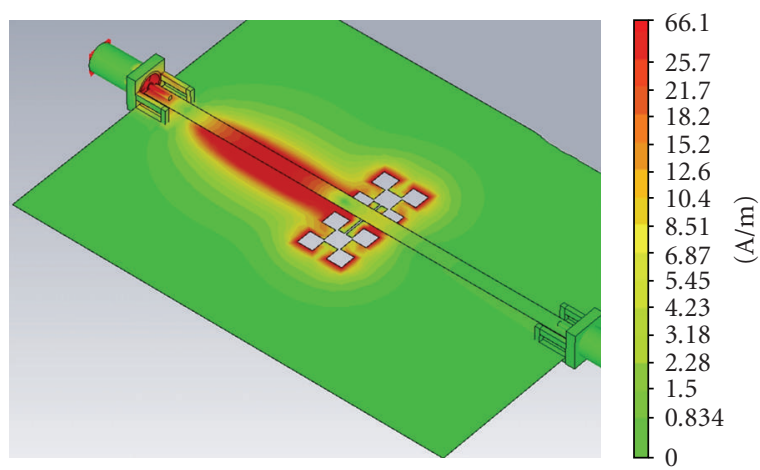

FIGURE 11: The proposed prefractal DGS cell acting on the wave launched down a microstrip line. The SCD was calculated at the resonance frequency, where the DGS is reflective and creats a standing wave pattern. Only copper parts are shown here; the dielectric substrate is hidden. The SCD plot shows concurrent maxima, which are physically unrealizable due to phase shifting.

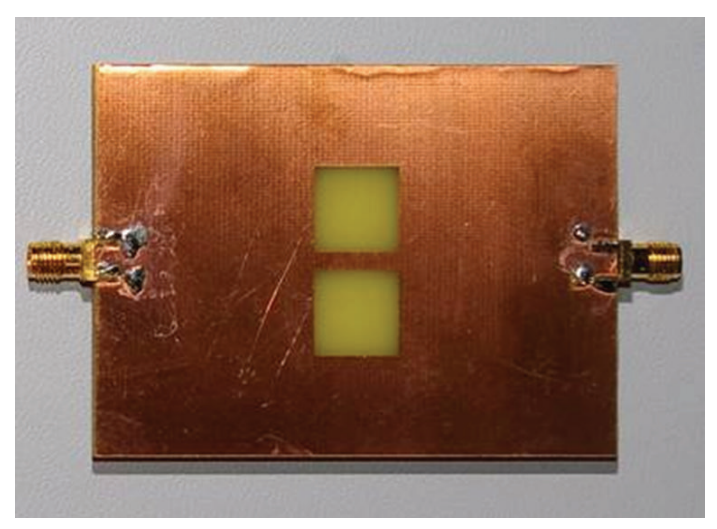

Figure 12: A standard dumbbell DGS etched on the Bottom layer of a microstrip circuit. The board measures $80 \mathrm{~mm} \times 60 \mathrm{~mm}$, whereas the size of the defect is $29.8 \mathrm{~mm} \times 13.5 \mathrm{~mm}$. The microstrip runs on the Top layer between the two connectors. The DGS comprises two square apertures connected with a thin slot $(0.3 \mathrm{~mm})$. Microstrip width equals the distance of the two apertures.

findings confirmed earlier simulation studies in that the length of the current path around the structure determines the distributed inductance, whereas the capacitance is mainly controlled by the width of the narrow slot that connects the two larger etched areas.

DGSs have been studied extensively for the past decade, and they have been successfully applied in the design of filters and amplifiers. Their versatility has also found use in the antenna field [40], though not nearly as much as in microstrip filters and amplifiers. The properties of the DGS as a resonator are given by:

$$
\begin{gathered}
Q=\frac{R}{\omega_{0} L}=\omega_{0} R C, \\
\mathrm{FBW}_{3 \mathrm{~dB}}=\frac{1}{Q},
\end{gathered}
$$

where $\omega_{0}$ is the resonance frequency, $Q$ is the quality factor, $\mathrm{FBW}_{3 \mathrm{~dB}}$ is the 3-dB fractional bandwidth of the stopband,

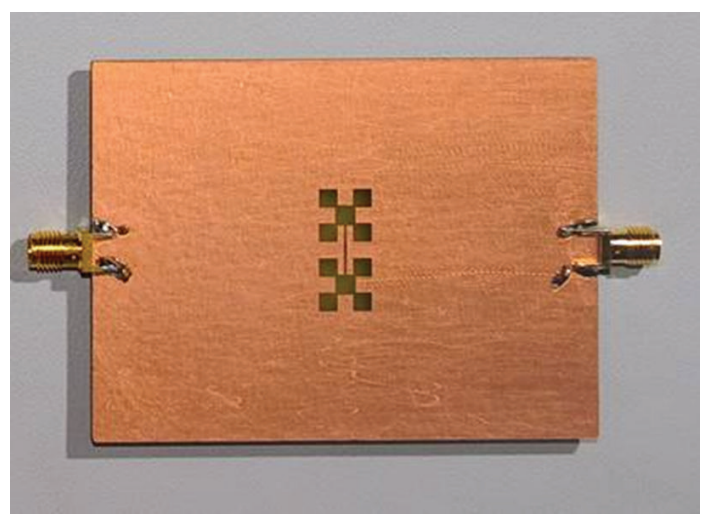

FIGURE 13: A prefractal dumbbell DGS etched on the Bottom layer of a microstrip circuit. The board measures $80 \mathrm{~mm} \times 60 \mathrm{~mm}$, while the size of the defect is $19.2 \mathrm{~mm} \times 8.2 \mathrm{~mm}$.

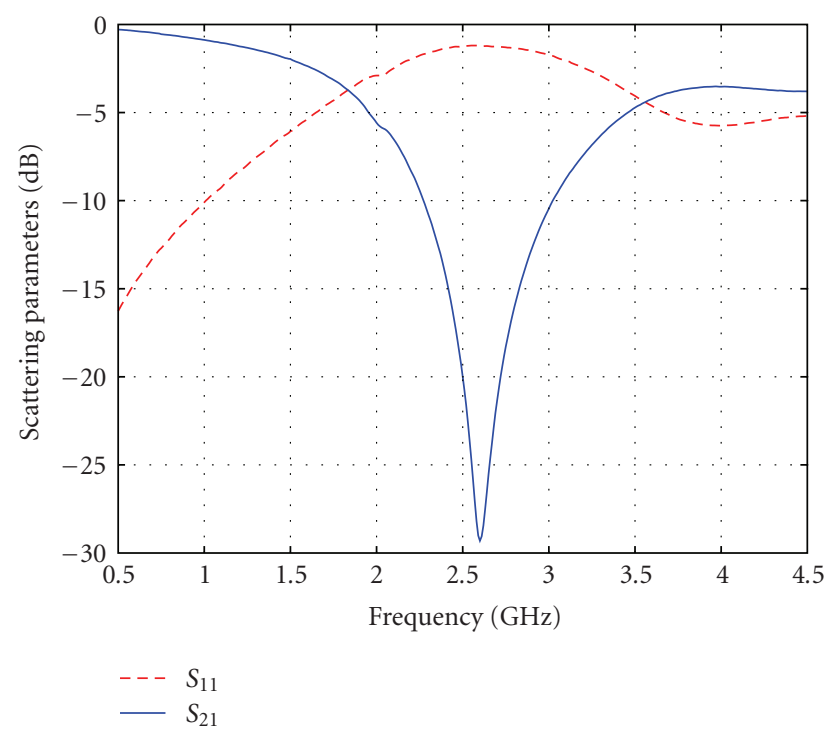

FIGURE 14: Measured S-parameters of the DGS-loaded microstrip line shown in Figure 13. The proposed DGS resonates at $2.6 \mathrm{GHz}$, causing high reflection and little signal transmission around that frequency.

and $R / L / C$ denote total distributed resistance, inductance, and capacitance at resonance.

The proposed DGS cell is shown in the model of Figure 11, where the defect has been etched below a microstrip line (the substrate has been removed for clarity). A picture of the actual circuit is shown in Figure 13. It is based on the dumbbell DGS, but it was eventually given a prefractal shape; it is a first-order Sierpinski carpet. Its overall dimensions are $19 \mathrm{~mm} \times 8 \mathrm{~mm}$. The measured electrical performance of the DGS is shown in Figure 14, where a wide stopband is formed around $2.6 \mathrm{GHz}$. In terms of signal transmission, the defect acts mainly as an open-circuit, which creates a standing-wave pattern across the line. It also acts partially as an antenna (wavetrap). The resonance is not very deep; this is a low- $Q$ structure. The quality factor was estimated from the $3-\mathrm{dB}$ fractional $\mathrm{BW}$ at $Q=1.3$. Nevertheless, this is just a blessing in disguise: the $30-\mathrm{dB}$ 


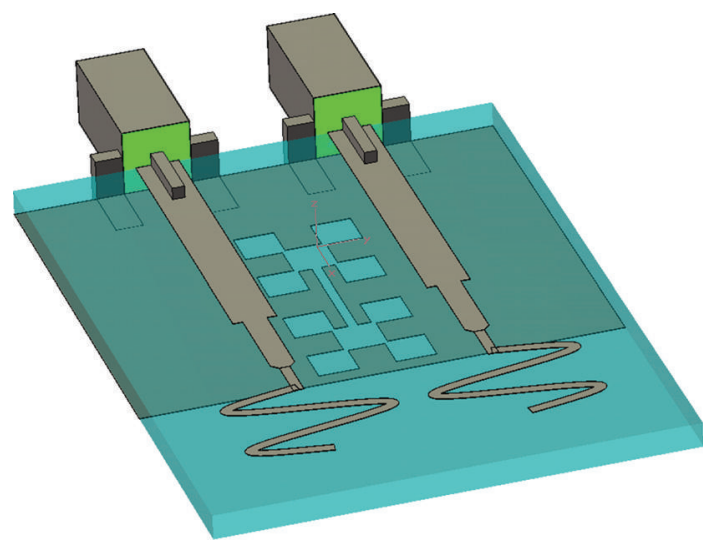

FIgURE 15: Perspective view of the 2-element compact array with embedded DGS. The FR4 substrate is transparent so that the defect on the GNDP is visible. In this snapshot, the interelement distance is $18 \mathrm{~mm}(0.15 \lambda)$.

rejection is more than enough for the reduction of mutual coupling, whereas the low $Q$-factor of the resonator makes the stopband wide enough to match the BW of the antenna. This is actually the reason why the defect was shaped after a prefractal: for a wider stopband, larger inductance and less capacitance are needed. A longer current path increases inductance significantly, thus enabling a wider connecting slot. Fractal shapes are a well-known quasideterministic method to increase wire length in a confined area and have been extensively applied to antennas [41].

A common misconception in DGS-related literature is that the frequency response relates to the area of the defect, for example, that resonance frequency is inversely proportional to area. This notion is repeated even in recent surveys on the topic [42]. The studied DGS models indicate that this is not so. It is the perimeter of the defect, rather than its area, that affects the frequency response. This is demonstrated briefly through a simple example. The prefractal defect in Figure 11 was transformed into a dumbbell-shaped defect that resonated at the same frequency $(2.5 \mathrm{GHz})$. All other parameters of the model remained the same as before. The area of the new defect was $24 \%$ larger, moving from 136 to $169 \mathrm{~mm}^{2}$. At the same time, the perimeter changed only by $4 \%$, increasing from 50 to $52 \mathrm{~mm}$.

3.2. DGS-Loaded Compact Antenna Array. The concept behind the proposed technique is to insert a properly resonating DGS cell between the two elements of the array and estimate the reduction in mutual coupling. As shown in Figure 15, the prefractal DGS is inserted (etched away) perpendicular to the flow of current between the two elements. It attempts to block the ground currents that contaminate the signal of the other antenna (signal bleeding). The PCB area occupied by the DGS decoupler is $17.3 \mathrm{~mm} \times 7.3 \mathrm{~mm}$, or $0.14 \lambda \times 0.06 \lambda$. The DGS cell is placed $1 \mathrm{~mm}$ away from the GNDP edge, where the return currents are stronger and increased self-inductance occurs.

The frequency response of the defected array in Figure 16 shows that the disturbance of the current caused by the DGS

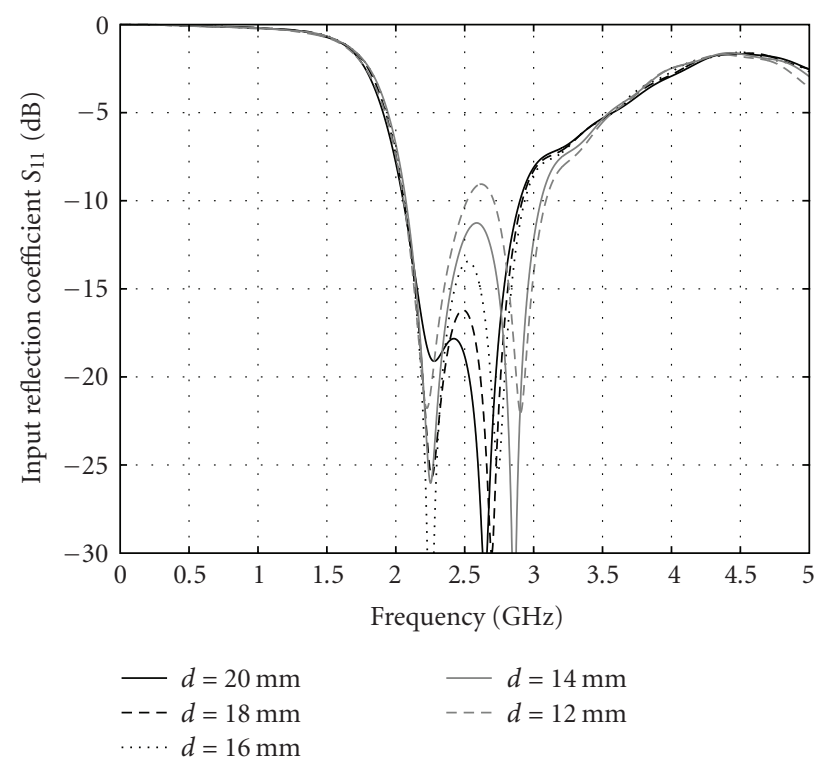

FIGURE 16: Parametric study of the broadband input matching of the DGS-loaded array for different values of interelement distance $(0.17 \lambda$ to $0.10 \lambda)$.

leads to a working antenna with a $1-\mathrm{GHz}$ operational BW. The interelement distance affects the input impedance, and this can be handled by resizing the elements and rematching at the feed point, but this is hardly the dominant factor here: the 2D surface current flowing across the GNDP is perturbed by the defect and more paths that are resonant are created. This is the reason behind the bandwidth expansion (or bandwidth recovery) seen in Figure 16 compared to Figure 6.

Nevertheless, the BW augment is just a pleasant side effect of the insertion of the defect. The design procedure opted for another sort of impact. Indeed, the current disturbance and the resonant behavior of the DGS offer a multidecibel drop in mutual coupling measured in terms of $S_{21}(j \omega)$ in Figure 17. For the $0.17 \lambda$ distance the drop is $10 \mathrm{~dB}$, while for the $0.10 \lambda$ distance more than $15 \mathrm{~dB}$ of coupling reduction were gained.

This reduction was also studied in terms of envelope correlation coefficient, which is an established metric for multielement antennas. The results in Figure 18 depict the broadband envelope correlation caused by the DGS when the two elements are spaced $\lambda / 10$ apart. The DGS that was applied is shown in Figure 19. The width of the long slot (nominally set at $0.6 \mathrm{~mm}$ ) is one of the crucial dimensions of the defect, because it controls the distributed capacitance.

Three different values of slot width were used. The study shows that as the slot widens, the defect becomes more "wideband"; that is, its stopband widens. Eventually, when the $0.6-\mathrm{mm}$ wide slot was used, the envelope correlation was practically eliminated inside the operating $\mathrm{BW}_{\mathrm{V}}$ of the antenna element. In this way, the DGS bandgap is translated into a mutual coupling bandgap and consequently into a correlation bandgap for the compact array.

Getting into the details of the mechanism behind coupling suppression, Figure 20 shows three snapshots of the current distribution on the ground plane. The snapshots 


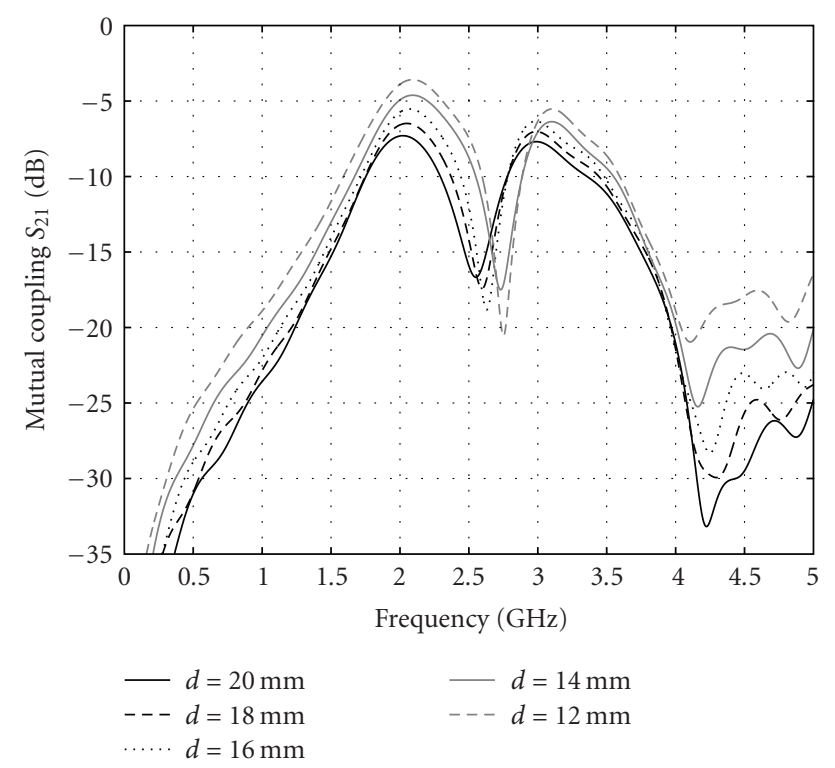

FIGURe 17: Parametric study of the broadband mutual coupling of the DGS-loaded array for different values of interelement distance $(0.17 \lambda$ to $0.10 \lambda)$.

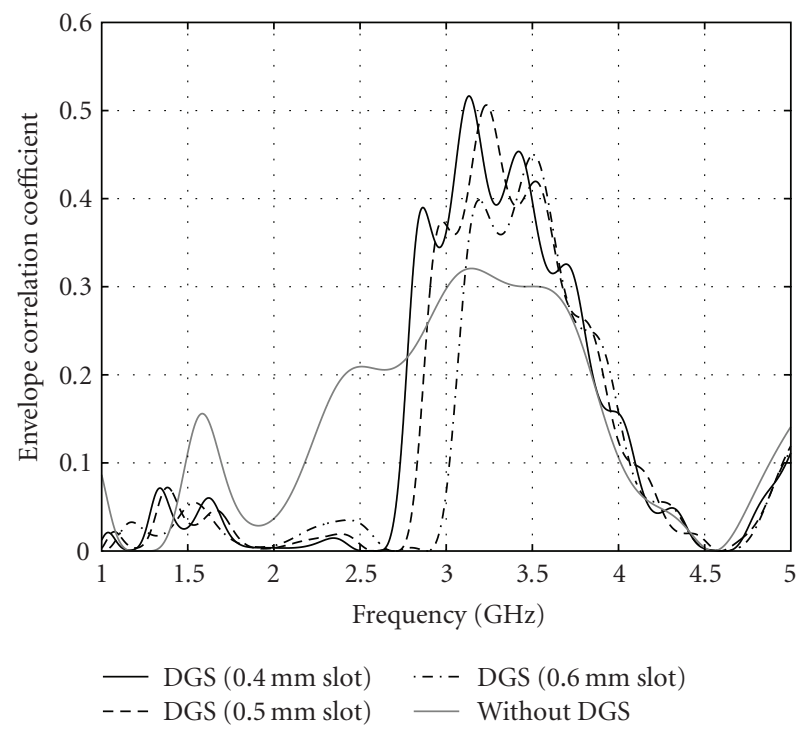

FIGURE 18: Significant reduction of the envelope correlation in the $2-3 \mathrm{GHz}$ range was achieved with the DGS at $0.10 \lambda$. The correlation remains high in the $3-4 \mathrm{GHz}$ range, but this is outside the operating BW of the array.

correspond to three different frequencies; the middle one lies inside the bandgap, while the other two are out-of-band. In all cases the left element $(\# 1)$ is excited, while element $\# 2$ is terminated at $50 \Omega$. The signal bleed into element \#2 is obvious outside the bandgap. Inside the stopband of the DGS, the resonance of the defect is evidenced by the increased current density across its vertices. The ground currents are partly trapped and radiate, but mostly they are reflected, and thus element \#2 stays unaffected.

3.3. Comparison of the DGS Decoupler with Other Simple Approaches. Following the paradigm set by Yang and

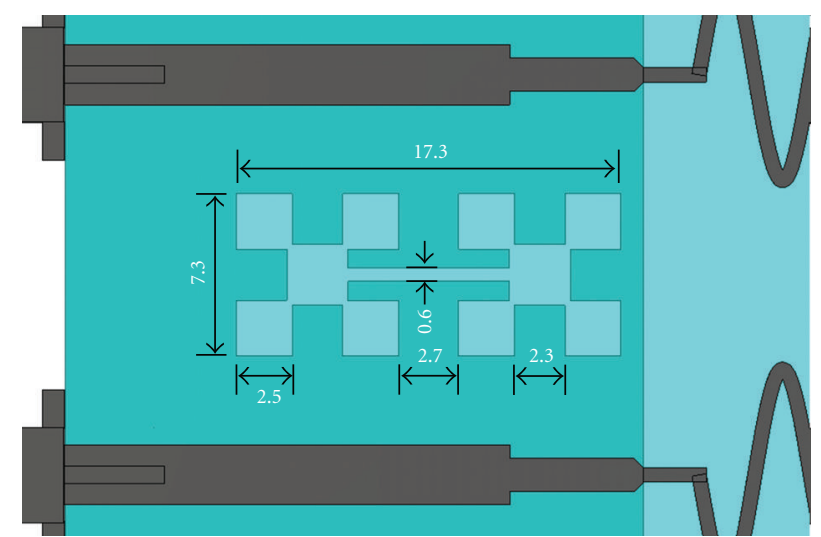

FIgURE 19: A closer look at the proposed PBG structure. The FR4 substrate is transparent so that the defect on the GNDP is visible. All dimensions are in millimeters. The overall PCB real estate occupied by the defect is $17.3 \mathrm{~mm} \times 7.3 \mathrm{~mm}$.

Rahmat-Samii [17], it is instructive to compare the DGS decoupling technique with other simple and low-cost generic techniques, such as,

(i) the removal of substrate between antennas,

(ii) cavity-backed printed antennas.

During the comparison, the size of antennas, substrate properties, and antenna spacing in all structures was kept the same as in the DGS case. In the first structure, the width of the removed substrate was $7.3 \mathrm{~mm}$, and the removal was done end-to-end. This width is chosen to be the same as the width of the defect. When the cavity structure is used, the distance between adjacent conductive walls was also set to $7.3 \mathrm{~mm}$. The cavity-backed array is depicted in Figure 21.

The mutual coupling results displayed in Figure 22 show that the other techniques had a minor effect on coupling in the band of interest. Compared to the initial array, the improvement seen was approximately $0.5-1.5 \mathrm{~dB}$. The DGS decoupler stands out with its $15-\mathrm{dB}$ reduction in coupling. However, the technique is yet to be perfected; an increase in the width of the stopband is needed.

3.4. Measurement Results and Discussion. The three 2element arrays shown in Figure 5 were rebuilt with a prefractal defect inserted between each pair of elements. This leads to the three arrays shown in Figure 23. The measurement setup was described in Section 2.1.

The response of the defected array in Figure 24 shows that the DGS-induced current disturbance leads to a working antenna with an operational $\mathrm{BW}_{\mathrm{V}}$ that rises to $1 \mathrm{GHz}$ and beyond (see Table 3). Much of the $\mathrm{BW}_{\mathrm{V}}$ of the singleelement antenna was recovered, but interelement distance affects the input impedance; the current footprint changes radically with IED due to the presence of the defect. The current disturbance and the resonant behavior of the DGS cell offer a multidecibel drop in mutual coupling measured in terms of $S_{21}(j \omega)$ : measured mutual coupling in Figure 25 shows $15-20 \mathrm{~dB}$ of reduction. The side effect of using 


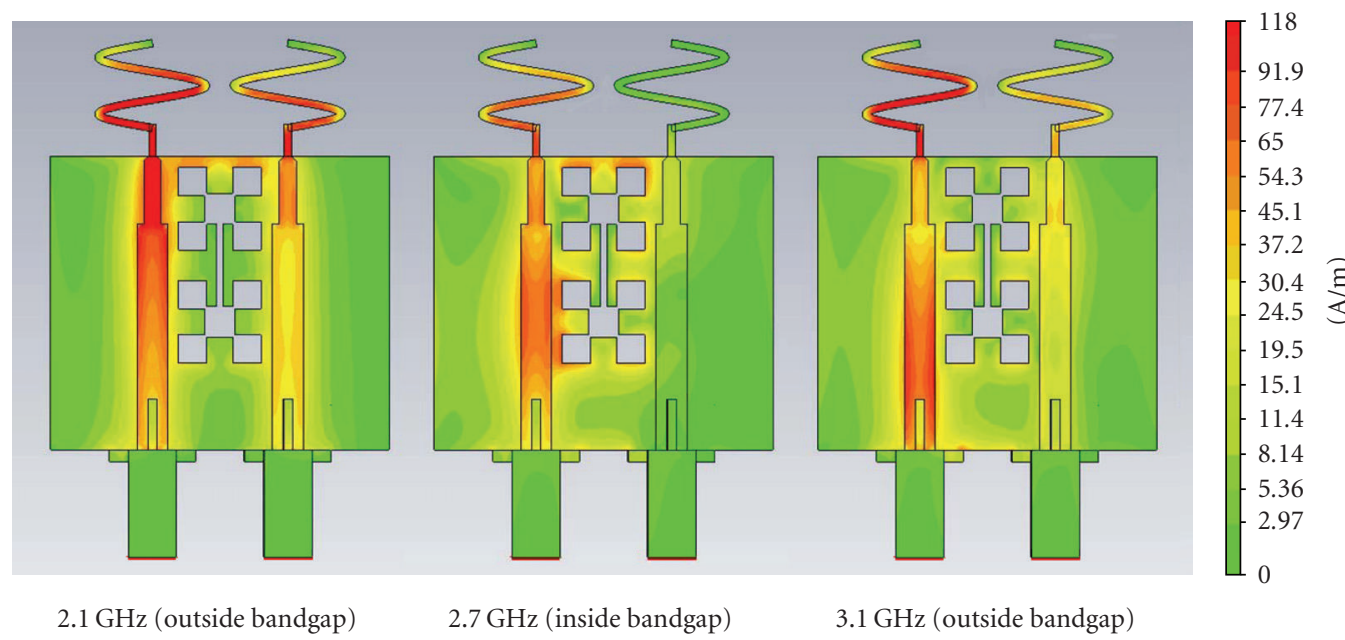

Figure 20: Port 1 is excited (left), while Port 2 is terminated at $50 \Omega$ (right). The surface current distribution on the conductive parts demonstrates the suppression of mutual coupling at $2.7 \mathrm{GHz}$.

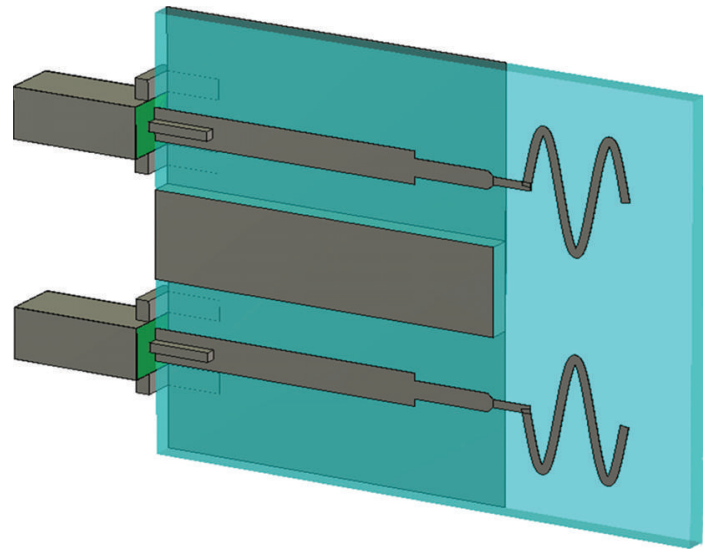

FIGURE 21: A two-element array that uses cavity backing instead of ground defect for coupling reduction.

a substrate with lower dielectric constant than the one assumed in simulations is obvious here: the defect resonated at $3 \mathrm{GHz}$ instead of $2.6 \mathrm{GHz}$. However, by comparison with the simulated data in Figure 16, it turns out that the substrate practically had no adverse effect on the input impedance results shown in Figure 24 . The reason is that $Z_{\text {in }}(j \omega)$ is mostly influenced by the element-GNDP coupling, that is, by the shapes, sizes, and SCDs of these two objects.

Finally, the envelope correlation coefficient shown in Figure 26, which was calculated from the measured complex $S$-parameters of the array with IED $d=18 \mathrm{~mm}$, remained below $\rho_{e}=0.2$ for all frequencies. This is even more pronounced in Figure 27, where for $\lambda / 10$ spacing the coefficient stays below $\rho_{e}=0.3$. The formation of a correlation bandgap in the $2-3 \mathrm{GHz}$ range is apparent.

By comparison with the results reported in recent literature [17-24], it is concluded that the three most important merits of the DGS decoupler are the small occupied PCB area, the width of the stopband (bandgap), and the resulting

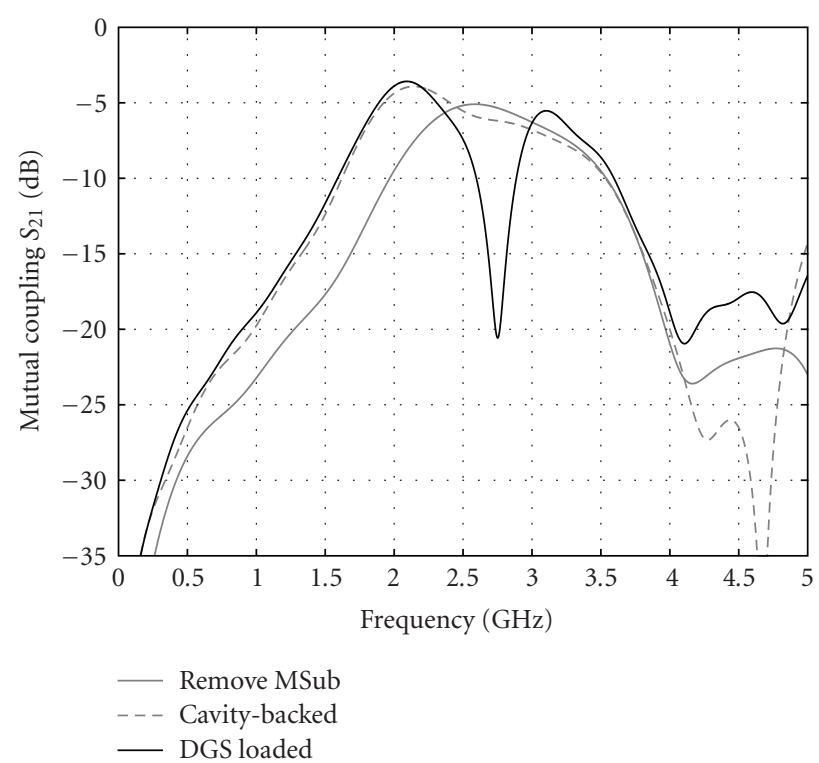

FIgURE 22: Comparison of mutual coupling reduction using different decoupling techniques.

active impedance bandwidth. A secondary advantage is the adjustable depth of resonance, which is controlled by balancing distributed inductance and capacitance. Regarding the occupied area, the results listed in Table 4 attest that the DGS decoupler requires much less real estate compared to previously proposed decouplers; in fact, the difference is such that the DGS method reduces the smallest area required by other methods by another $60 \%[19,21]$.

Regarding the two bandwidths, a few comments are in order. First, decoupling methods are applied at either the circuit level or the antenna level. Circuit-level decoupling, which tries to solve the multiport-conjugate-matching problem [24], is well outside the scope of this paper. An example circuit decoupler is the transmission line network presented in [18]. Antenna-level decoupling can be done 


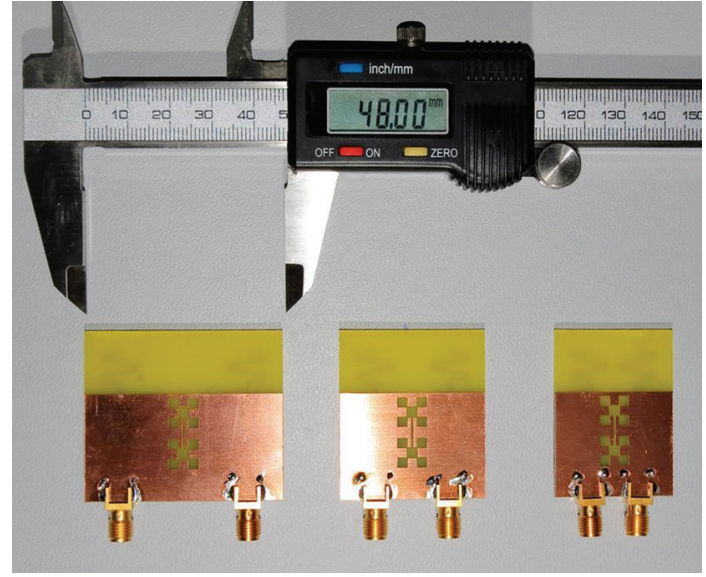

FIGURE 23: The bottom layer of three fabricated DGS-loaded compact arrays. From left to right, the interelement distance is $30 \mathrm{~mm}(0.25 \lambda), 18 \mathrm{~mm}(0.15 \lambda)$, and $12 \mathrm{~mm}(0.10 \lambda)$, respectively.

TABLE 3: Active input impedance bandwidths of the three measured DGS-loaded arrays.

\begin{tabular}{lccc}
\hline$d(\mathrm{~mm})$ & Center frequency $(\mathrm{GHz})$ & $\mathrm{BW}_{\mathrm{V}}(\mathrm{GHz})$ & $\mathrm{FBW}_{\mathrm{V}}(\%)$ \\
\hline 30 & 2.575 & 0.952 & 37.0 \\
18 & 2.568 & 1.027 & 40.0 \\
12 & 2.573 & 1.145 & 44.5 \\
\hline
\end{tabular}

TABle 4: Comparison of occupied PCB real estate between proposed decoupling methods.

\begin{tabular}{lcc}
\hline Ref. \# & Size & Area $\left(\lambda^{2}\right)$ \\
\hline$[17]$ & $1.07 \lambda \times 0.30 \lambda$ & 0.3210 \\
{$[18]$} & $1.43 \lambda \times 1.34 \lambda$ & 1.9162 \\
{$[19]$} & $0.33 \lambda \times 0.07 \lambda$ & 0.0231 \\
{$[20]$} & $0.25 \lambda \times 0.14 \lambda$ & 0.0350 \\
{$[21]$} & $0.18 \lambda \times 0.12 \lambda$ & 0.0216 \\
{$[22]$} & $0.56 \lambda \times 0.11 \lambda$ & 0.0616 \\
{$[23]$} & $0.60 \lambda \times 0.20 \lambda$ & 0.1200 \\
This work & $0.14 \lambda \times 0.06 \lambda$ & 0.0084 \\
\hline
\end{tabular}

in three ways: modified ground plane, neutralization line, and parasitic scatterer [24]. All three techniques require that the structure of the array be modified. With the exception of [18], all other methods listed in Table 4 are modifiedground-plane decouplers. Modification of the GNDP has the obvious drawback that all subcircuits share ground and thus significant changes may not be feasible. As it was pointed out in [24], antenna-level decoupling methods generally suffer from two side effects: (a) tens of decibels of MC suppression are achievable, but only for a small fractional bandwidth, and (b) as the array elements draw closer, the active impedance bandwidth of each element diminishes. By looking at the reflection and coupling results in Figures 16, 17, 24, and 25, it occurs that the DGS decoupler does not face the above restrictions; hence, it provides for a promising antenna-level decoupling technique, once it is perfected.

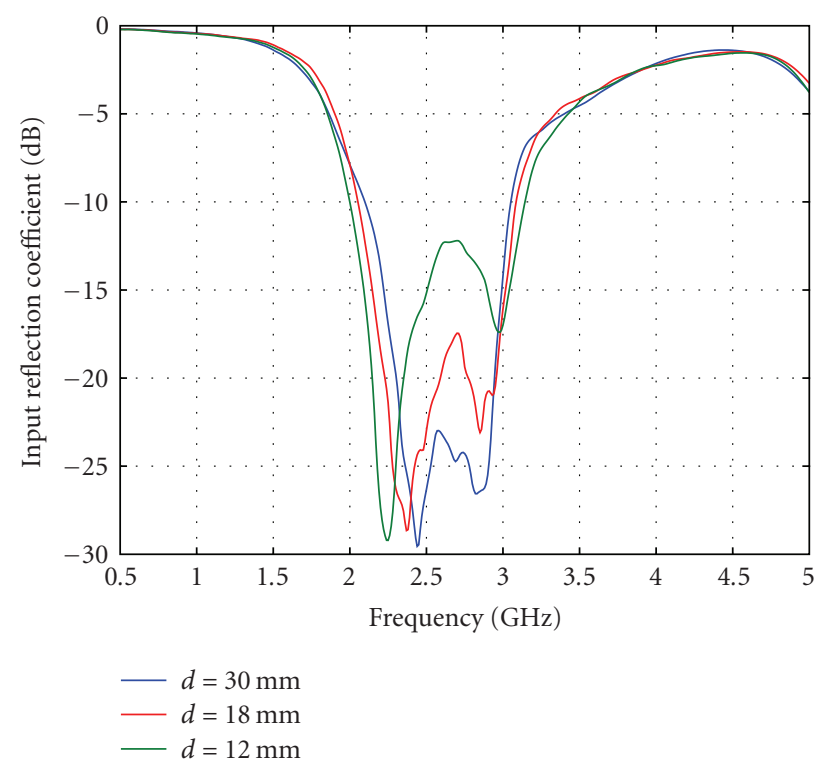

FIGURE 24: Measured active reflection coefficient and impedance bandwidth of the three 2-element arrays shown in Figure 23.

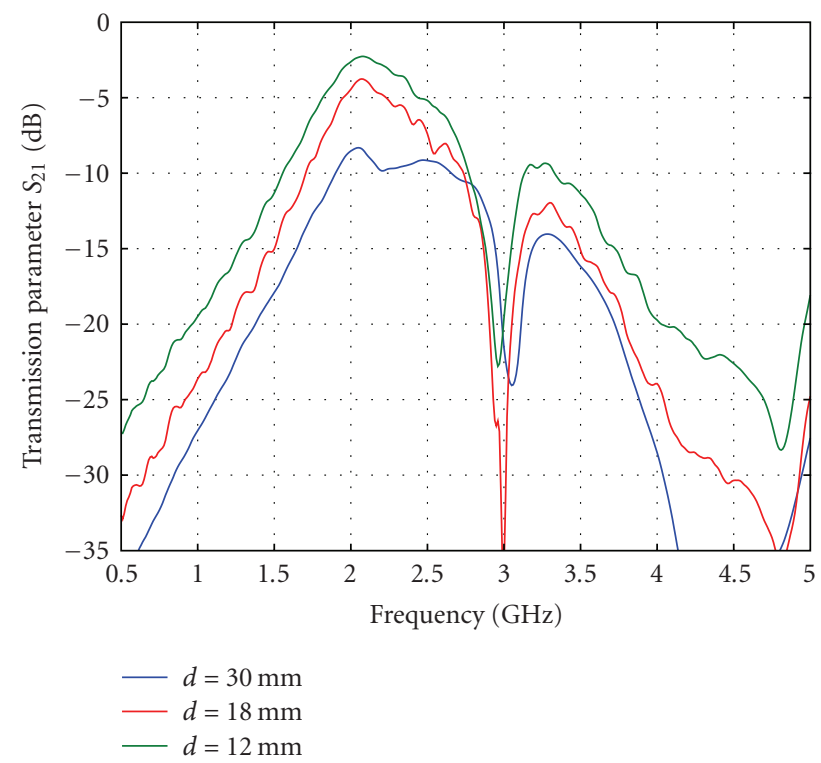

FIGURE 25: Measured mutual coupling between the elements of the arrays shown in Figure 23. Coupling was expressed in terms of $\left|S_{21}(j \omega)\right|$.

\section{Radiation Properties of the Two-Element Compact Array}

This section investigates the effect that both the problems discussed earlier and their solutions had on the radiation of the sinusoidal elements of the array. This was carried out by calculating the degradation of total efficiency due to the coupling, and by examination of the far-field patterns. To facilitate the evaluation of these results, the far-field of a single third-iteration monopole is quickly examined first. 


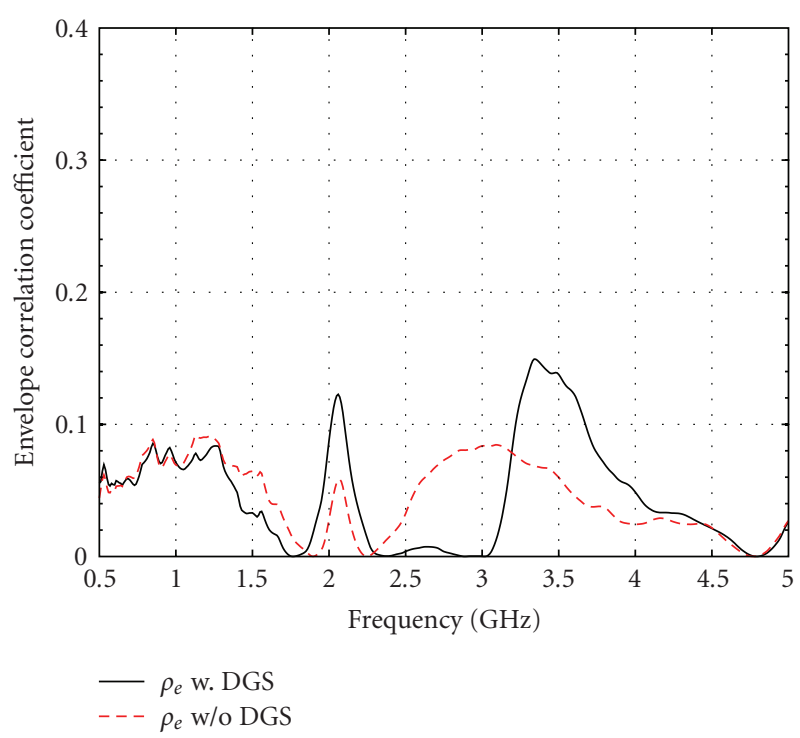

FIGURE 26: Broadband envelope correlation coefficient calculated from the measured complex $S$-matrix of the arrays with spacing $d=$ $18 \mathrm{~mm}=0.15 \lambda$.

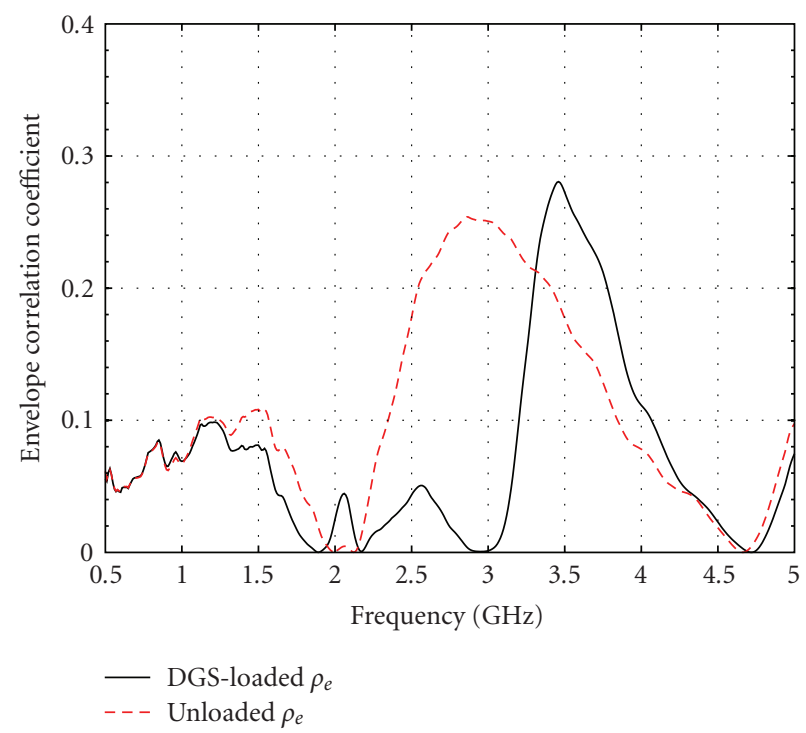

FIGURE 27: Broadband envelope correlation coefficient calculated from the measured complex $S$-matrix of the arrays with spacing $d=$ $12 \mathrm{~mm}=0.10 \lambda$.

The numerical 3D gain pattern (IEEE) shown in Figure 28 has been calculated at resonance and indicates that the printed structure radiates in dipole mode. It exhibits the well-known toroidal pattern, which, in this case, shows stronger radiation towards the backside of the PCB, because of the presence of the ground plane. The radiation pattern actually corresponds to a thick asymmetric dipole. The achievable gain was estimated at $G_{\max }=2.6 \mathrm{dBi}$, reaching up to $0.5 \mathrm{~dB}$ higher than the $\lambda / 2$-dipole. Part of this excess gain comes from the strong currents on the GNDP; the SCD occupies a larger volume inside the circumscribing sphere compared to a thin wire dipole. The rest is due to the

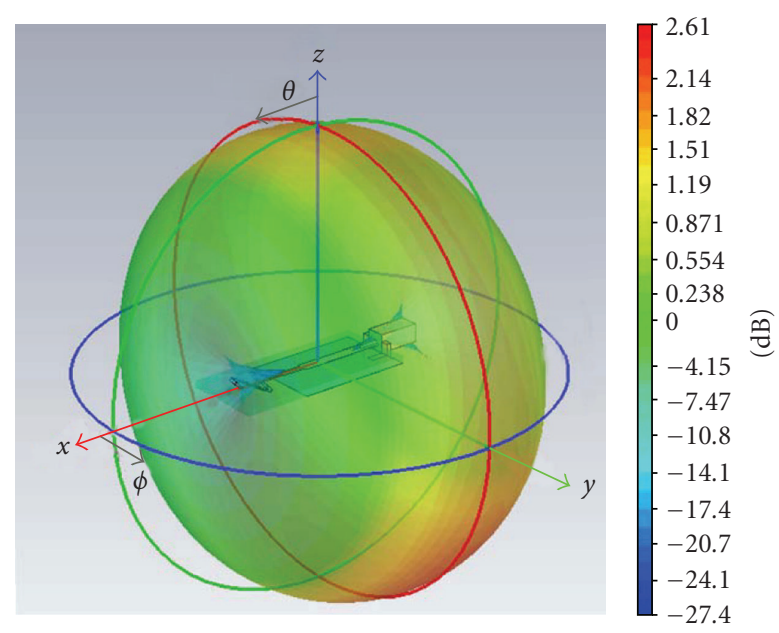

FIGURE 28: Transparent 3D far-field embedded around the $i=$ 3 antenna. The far-field pattern was calculated at the $2.5-\mathrm{GHz}$ resonance.

currents flowing on the outer shield of the coaxial connector; these currents artificially increase the electrical size of the overall radiator.

The omnidirectional gain pattern shown in Figure 28 is very desirable for small portable terminals, such as wireless sensor nodes. These terminals often operate in a richscattering environment, where incoming waves arrive from all directions in space; that is, they have a large angular spread $[25,29-31]$. In this case, terminals need to be able to receive efficiently from all directions. The $3 \mathrm{D}$ pattern shows that reception problems occur only along the $x$-axis.

Starting with the initial (unloaded) array, the results in Figure 29 reveal the gradual degradation of total efficiency as the elements were packed closer. The data were extracted by feeding the input port of one of the elements while terminating the other port at $50 \Omega$. In this configuration (6) estimates the total efficiency:

$$
n_{\text {total }}=n_{\text {rad }}\left(1-\left|S_{11}\right|^{2}-\left|S_{21}\right|^{2}\right) .
$$

When the elements were spaced at $d_{\max }=\lambda / 4$, there was moderate mutual coupling, and thus total efficiency stayed above $70 \%$. When the spacing dropped to $d_{\min }=\lambda / 8$, efficiency dropped below $60 \%$ and even as low as $45 \%$. The loss in antenna efficiency is particularly problematic for the energy efficiency of a sensor node. Figure 30 depicts one of the active element patterns of the array [32]. It is evident that the directions of maximum gain have been rotated due to the presence of the parasitic second element and the wider GNDP. Reception from all directions in space was maintained. The elements are too close $(d=15 \mathrm{~mm}=\lambda / 8)$ to provide increased directivity in any direction.

By inserting the resonating defect between the two elements, the situation with total efficiency improved significantly. This is evidenced by the efficiency curves in Figure 31 . At every distance, and within a certain band, efficiency was largely recovered. The DGS cell provided $n_{\text {total }}>60 \%$ even for $\lambda / 10$ spacing. The defect can be tailored to the designer's needs. A change in dimensions would alter the resonance 


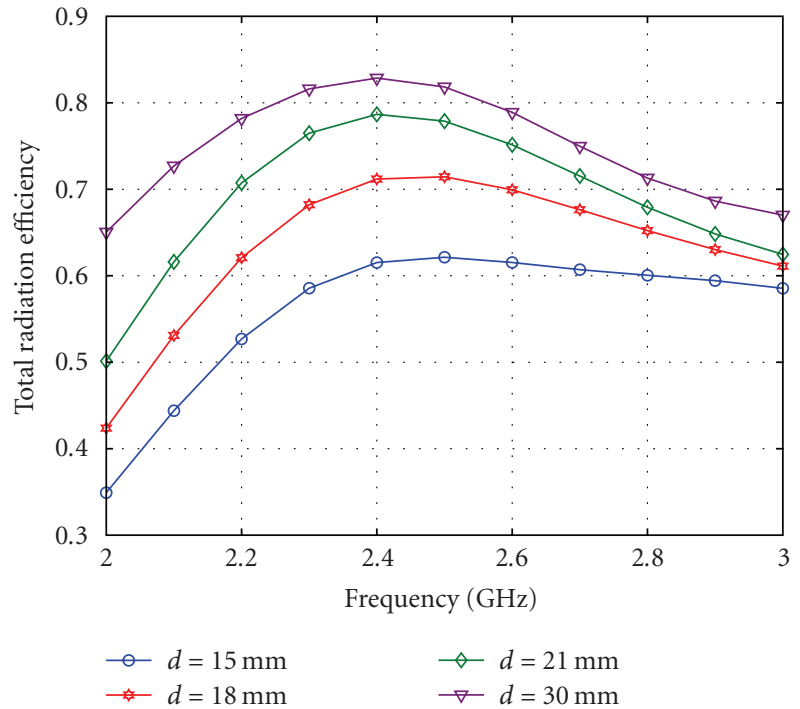

FIgURE 29: Variation in total efficiency with variable IED in the 2$3 \mathrm{GHz}$ range for the initial compact antenna array.

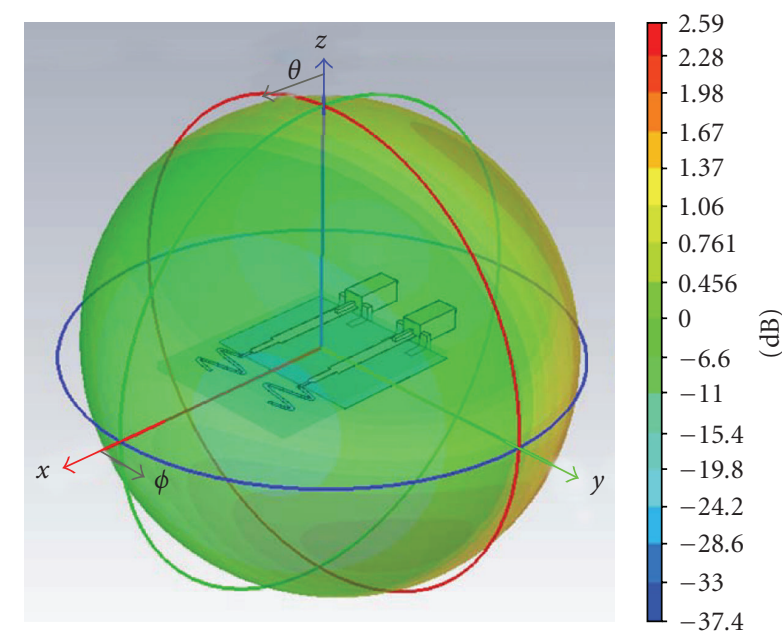

Figure 30: The transparent 3D active element pattern of the initial array embedded around the sensor node. The pattern was calculated at $2.5 \mathrm{GHz}$ and corresponds to excitation of the right element.

and thus improve the efficiency in any specific desired band. Another open problem is the possible distortion of the radiation pattern in the far-field: the defect acts partly as a wave trap and radiates. However, as shown in Figure 32, the ground defect is an inefficient "slot antenna" and practically does not affect the pattern, which shows very little change when compared with the case of no DGS loading. The directions of maximum radiation rotate slightly towards the $\theta=0$ axis, and maximum gain values increased by $\delta G_{\max }=$ $+0.1 \mathrm{~dB}$.

\section{Conclusions}

Energy efficiency is an important topic in wireless sensor networks and an important metric that sets them apart from

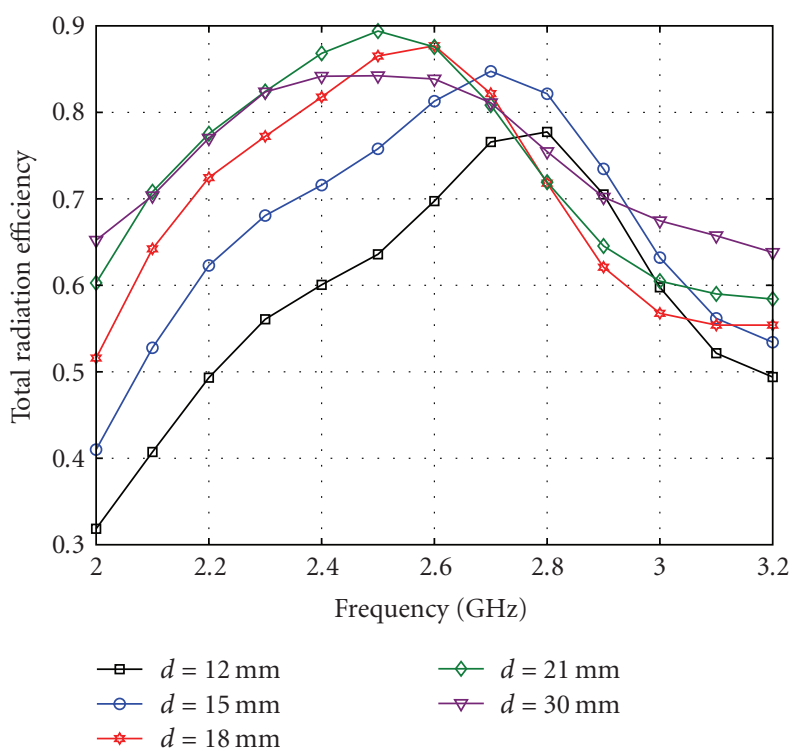

FIGURE 31: Variation in total efficiency with variable IED in the 2$3 \mathrm{GHz}$ range for the DGS-loaded compact antenna array.

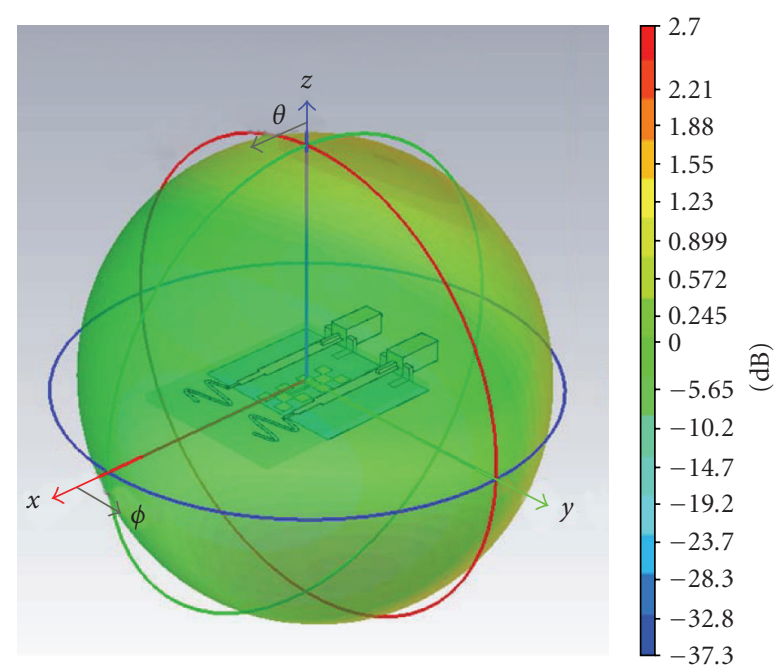

FIGURE 32: The transparent 3D active element pattern of the DGSloaded array embedded around the sensor node. The pattern was calculated at $2.5 \mathrm{GHz}$ and corresponds to excitation of the right element.

typical ad hoc networks. The goal of WSN system design is to maximize the lifetime of the network, that is, to maintain a reasonable level of connectivity for the largest possible duration. Wireless communication circuits tend to dominate the system energy budget [2]. The antenna is the spearhead of the RF front-end; thus WSN-targeted antennas should be designed with efficiency in mind.

Sinusoidal monopoles integrate well with portable devices. Sinusoids function well with relatively small GND planes $(\sim \lambda / 4 \times \lambda / 6)$ and thus provide for building compact printed arrays. Some detuning occurred, although not severe and easily compensated by following a few guidelines (see Appendix A). The frequency response showed graceful 
degradation with denser interelement spacing. What is more, extreme values of mutual coupling did not lead to extreme signal correlation.

The sinusoidal elements, the GNDP size effect, and the two-element array were backed by measurements on actual hardware. Inaccurate assumptions regarding the properties of available FR-4 substrates caused testing of devices on materials with lower permittivity at $2.5 \mathrm{GHz}$. Still, the measured antennas displayed a propensity for large VSWR bandwidths despite their small size. Even in their current implementation, sinusoids offer the ability to trade excess bandwidth for further size reduction.

Eventually CMOS-integrated circuits will advance to the point of enabling diversity (and perhaps even MIMO) applications on tiny sensor nodes. Antenna technology can keep up with this trend so long as PCB real estate and size are judiciously exploited. In any case, sensor radios face a challenging environment; so architectures with robustness to deep fading are required [2]. To this end, the insertion of a single DGS cell in the ground plane of a generic 2element array reduced mutual coupling inside the band of interest and formed a correlation bandgap. The proposed technique is cost-effective since it is compatible with $\mathrm{PCB}$ printing processes and offers multidecibel gains in coupling and correlation reduction. Finally, it was shown that the defect is an inefficient slot antenna, and so the distortion of the radiation pattern was avoided.

Taking a number of well-performing single antenna elements and displacing them as far as possible will not suffice for future compact multi-element antennas. A more rigorous and holistic approach is needed to achieve good overall array characteristics. This is a very challenging task when the combination of elements and GNDP becomes electrically small [24]. So, further work is needed on the defect itself: it has to be made electrically smaller to enable closer antenna packing. Dielectric loading with a material of higher dielectric constant must be avoided, because it would compromise antenna efficiency. It would also be very useful to discover ways to make its stopband even more wideband. To these ends, other prefractal and Euclidean topologies will be investigated. The compensation of array element detuning is yet to be demonstrated; it was omitted here for brevity. Finally, an extension of this technique to four-element compact arrays is planned, which would be targeted at more complex systems such as WiFi routers. Fourelement arrays are much more appealing to MIMO system applications, because they can extract the full rank of a realistic environment that is rich in scatterers [43].

\section{Appendices}

\section{A. Printed Sinusoidal Antenna Design Guide}

Figure 33 depicts all significant parameters that control the design of the antenna. The sinusoidal curve is characterized by its amplitude A and spatial period $P$. The initial straight segment is crucial to the operation of the antenna: it drives the first horizontal segment away from the GNDP and improves radiation efficiency. Its length was set equal to

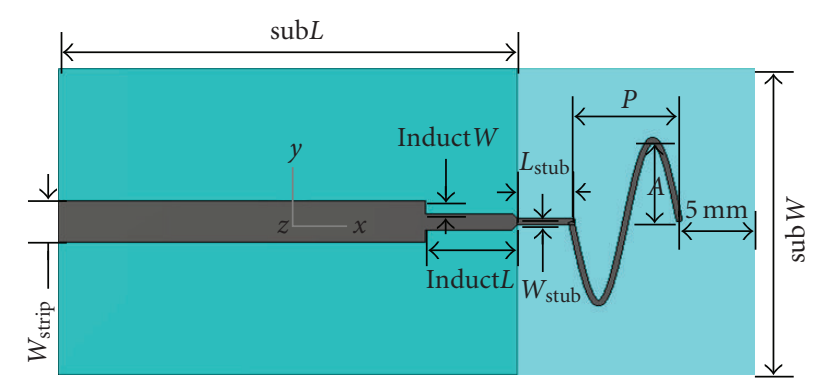

FIgURE 33: The top layer of the fully parameterized sinusoidal "system model". The element is a "second iteration sinusoidal monopole" $(i=2)$. The substrate material is transparent so that the ground plane on the Bottom layer is visible. Element size is $2 A \times 2 A \mathrm{~mm}^{2}$.

a half-period $\left(L_{\mathrm{stub}}=P / 2\right)$, which is adequate but by no means optimal; this is a subject of future work. The design is very repeatable, since it is based on an analytical curve. The copper segments that produce the antenna element are formed by $(x, y)$ points obeying the parametric description of (A.1), in which $\xi$ is the independent parameter:

$$
\begin{gathered}
x=\xi, \\
y=-A \sin \left[\frac{2 \pi}{P}\left(\xi-\frac{P}{2}-\frac{\operatorname{sub} L}{2}\right)\right] .
\end{gathered}
$$

Numerical studies revealed that the input impedance $Z_{\text {in }}(j \omega)$ of the sinusoidal antenna is capacitive, as is typical of meander-line structures. This effect was exploited by narrowing the last segment of the microstrip, which served as an inductor in series with $Z_{\text {in }}(j \omega)$; it is the simplest matching network. The length of this "lumped element" is $18^{\circ}$ at $2.5 \mathrm{GHz}$. The main point in the design guide of these antennas is that the series inductor can be combined with the width of the element $W_{\text {stub }}$ to shape the input reflection coefficient in the frequency domain and thus tailor the bandwidth to the designer's needs.

It is a trivial optimization task to show that minimum "antenna spread" on the PCB is achieved when the outline, or "envelope", of the element becomes square. Since sinusoids are folded monopoles, their unfolded length is constrained to be somewhat greater than $\lambda / 4$. This constraint imposes an equivalent constraint on the sum of the two sides of the envelope, because of spatial uniformity. Turning the envelope into a square produces the envelope with the shortest diagonal, hence the smallest spread on the PCB. The constraint in (A.2) was applied to the dimensions of the sinusoidal antenna to enforce the square spatial envelope:

$$
2 A=\frac{P}{2}(i+1) .
$$

The procedure to obtain a working sinusoidal antenna of given iteration $i$ is summarized in the following design guide.

(i) Starting from a reasonable value of $W_{\text {stub }}(1-2 \mathrm{~mm})$ and zero series inductance, the simulator calculates the spatial amplitude A that produces a lower resonance at the desired frequency $f_{0}$. This calculation 
can be either a parametric sweep or an optimization. The optimization is single-parameter/singlegoal; thus the optimizer should take a reasonable amount of time to converge. An initial value for the spatial amplitude would be $A=\lambda /(10 i)$.

(ii) The value of inductW is altered to optimize the input reflection coefficient in the desired manner. For example, the input capacitance can be cancelled up to the point where the $\Gamma_{\text {in }}$ curve is distributed evenly about the real axis of the Smith Chart near the resonance.

(iii) The value of $W_{\text {stub }}$ is decreased to the point where the antenna cannot provided the desired impedance bandwidth. If the trace is sufficiently wide, two dominant current paths exist, producing a dual-mode antenna. Below a certain $W_{\text {stub }}$ threshold, only one mode is supported and bandwidth drops. Parameter $W_{\text {stub }}$ is a miniaturization technique embedded in the antenna itself: decreasing $W_{\text {stub }}$ leads to decreased amplitude $\mathrm{A}$ for a given resonance, and thus to smaller element size, albeit with reduced bandwidth.

(iv) If the choice of $W_{\text {stub }}$ leads to an electrically smaller element (lower $f_{0}$ ), amplitude A can be readjusted.

\section{B. Simulation Setup}

The antennas were designed and simulated in a reliable Transient Solver [44], which exhibited good correlation between simulated and measured results in prior studies [29-31]. The Solver is part of a full-wave electromagnetic simulator that applies the Finite Integration Technique (FIT) to reformulate Maxwell's integral equations into the so-called "Maxwell Grid Equations". In the time domain, by applying Yee's spatial discretization and time-stepping scheme, FIT results in the same set of equations as FDTD.

A wideband Gaussian pulse excited the structures (DC$5 \mathrm{GHz}$ ). A spatially adaptive hexahedral mesh discretized the objects. Finer meshing was applied inside the substrate to capture the large gradients of the E-field; the same applies across the microstrip feed, on the sinusoidal element, and inside the dielectric of the SMA connector. The simulator stopped when the initial system energy decayed by $50 \mathrm{~dB}$. This was a good trade-off between simulation speed and truncation error in the FFT engine that translates the results from the time- to the frequency-domain. The maximum cell size at the maximum frequency (smallest wavelength) was set at $\lambda_{5-\mathrm{GHz}} / 25$. The solvable space was terminated at several Berenger PML layers.

(i) During the initial design stages, four layers were used to speed up the design cycle, with distance-toboundary equal to $\lambda_{2.5-\mathrm{GHz}} / 8$.

(ii) For the final simulations, six layers were used, with distance-to-boundary equal to $\lambda_{2.5-\mathrm{GHz}} / 4$. By increasing the distance to $\lambda_{2.5-\mathrm{GHz}} / 2$ the results did not improve further.

Whenever a model featured topological symmetry and satisfied the appropriate boundary conditions for electric and magnetic flow, a magnetic wall was placed across the plane of symmetry. This boundary condition cut the computational burden in half, because only half of the structured needed solving. The complexity of the models ranged between 250, 000 and 650, 000 Yee cells. Complexity depends upon the size of the GND plane and the level of detail exhibited by the antenna element. Narrow copper traces and narrow object spacing contribute greatly to complexity.

To reduce the computational complexity, a squared equivalent connector replaced the realistic model of the cylindrical end-launch SMA [25-28]. It is important to model the feed structure as accurately as possible without severely compromising simulation speed; the presence of the SMA submodel is necessary to get an accurate simulated surface current distribution. Thus, the SMA submodel helps predict the input impedance of the antenna with increased accuracy.

\section{References}

[1] B. Warneke, M. Last, B. Liebowitz, and K. S. J. Pister, "Smart dust: communicating with a cubic-millimeter computer," Computer, pp. 44-51, 2001.

[2] B. W. Cook, S. Lanzisera, and K. S. J. Pister, "SoC issues for RF smart dust," Proceedings of the IEEE, vol. 94, no. 6, pp. 11771196, 2006.

[3] P. Basset, F. Alfaro, D. Novosel, A. De La Plaza, D. Stancil, and G. K. Fedder, "Chip-size antennas for implantable sensors and smart dust," in Proceedings of the 13th International Conference on Solid-State Sensors and Actuators and Microsystems (TRANSDUCERS '05), pp. 457-460, Seoul, South Korea, June 2005.

[4] M. Haenggi and D. Puccinelli, "Routing in ad hoc networks: a case for long hops," IEEE Communications Magazine, vol. 43, no. 10, pp. 93-101, 2005.

[5] N. Heo and P. K. Varshney, "Energy-efficient deployment of intelligent mobile sensor networks," IEEE Transactions on Systems, Man, and Cybernetics, Part A, vol. 35, no. 1, pp. 7892, 2005.

[6] S. Jain, R. C. Shah, W. Brunette, G. Borriello, and S. Roy, "Exploiting mobility for energy efficient data collection in wireless sensor networks," Mobile Networks and Applications, vol. 11, no. 3, pp. 327-339, 2006.

[7] G. Manes, R. Fantacci, F. Chiti, et al., "Efficient MAC protocols for wireless sensor networks endowed with directive antennas: a cross-layer solution," EURASIP Journal on Wireless Communications and Networking, vol. 2007, Article ID 37910, 9 pages, 2007.

[8] J. Dunlop and J. Cortes, "Co-design of efficient contention MAC with directional antennas in wireless sensor networks," in Proceedings of the 13th International Wireless Communications and Mobile Computing Conference (IWCMC '08), pp. 383-388, Crete Island, Greece, August 2008.

[9] D. Li, Z. Li, and L. Liu, "Energy efficient broadcast routing in ad hoc sensor networks with directional antennas," in Proceedings of the Water and Sewerage Authority (WASA '08), vol. 5258 of Lecture Notes in Computer Science, pp. 29-39, 2008.

[10] Z. Li and D. Li, "Minimum energy broadcast routing in ad hoc and sensor networks with directional antennas," in Proceedings of the Computations in Commutative Algebra, vol. 5573 of Lecture Notes in Computer Science, pp. 507-518, 2009. 
[11] S. Shankar and D. Kundur, "Towards improved connectivity with hybrid uni/omni-directional antennas in wireless sensor networks," in Proceedings of the IEEE INFOCOM Workshops, Phoenix, Ariz, USA, April 2008.

[12] A. Lecointre, D. Dragomirecu, P. Berthou, and R. Plana, "A reconfigurable IR-UWB radio interface, with directional antennas and localization capability, for wireless sensor networks," in Proceedings of the 14th IEEE International Conference on Electronics, Circuits and Systems (ICECS '07), pp. 1055-1058, Marrakech, Morocco, December 2007.

[13] G. Giorgetti, A. Cidronali, S. K. S. Gupta, and G. Manes, "Exploiting low-cost directional antennas in $2.4 \mathrm{GHz}$ IEEE 802.15.4 wireless sensor networks," in Proceedings of the 10th European Conference on Wireless Technologies (ECWT'07), pp. 217-220, Munich, Germany, October 2007.

[14] M. Cabedo, M. Gallo, E. Antonino, M. Ferrando, and M. Bozzetti, "Modal analysis of a MIMO antenna for sensor networks," in Proceedings of the IEEE Antenna and Propagation Society International Symposium (AP-S '08), San Diego, Calif, USA, July 2008.

[15] A. M. Abbosh and D. V. Thiel, "Effect of mutual coupling on the energy requirement of MIMO-based wireless sensor networks," in Proceedings of the IEEE International Conference on Signal Processing and Communications (ICSPC '07), pp. 436-439, Dubai, UAE, November 2007.

[16] A. Marousis and P. Constantinou, "Performance of a low complexity channel estimator for MC-CDMA systems over realistic MIMO channels," in Proceedings of the 6th Conference Telecommunications, Peniche, Portugal, May 2007.

[17] F. Yang and Y. Rahmat-Samii, "Microstrip antennas integrated with electromagnetic band-gap (EBG) structures: a low mutual coupling design for array applications," IEEE Transactions on Antennas and Propagation, vol. 51, no. 10, pp. 2936-2946, 2003.

[18] S. Dossche, S. Blanch, and J. Romeu, "Optimum antenna matching to minimise signal correlation on a two-port antenna diversity system," Electronics Letters, vol. 40, no. 19, pp. 1164-1165, 2004.

[19] C.-Y. Chiu, C.-H. Cheng, R. D. Murch, and C. R. Rowell, "Reduction of mutual coupling between closely-packed antenna elements," IEEE Transactions on Antennas and Propagation, vol. 55, no. 6, pp. 1732-1738, 2007.

[20] T. Kokkinos, E. Liakou, and A. P. Feresidis, "Decoupling antenna elements of PIFA arrays on handheld devices," Electronics Letters, vol. 44, no. 25, pp. 1442-1444, 2008.

[21] T. Ohishi, N. Oodachi, S. Sekine, and H. Shoki, "A method to improve the correlation coefficient and the mutual coupling for diversity antenna," in Proceedings of the IEEE Antennas and Propagation Society International Symposium, pp. 507510, Washington, DC, USA, July 2005.

[22] M. Schühler, R. Wansch, and M. A. Hein, "Reduced mutual coupling in a compact antenna array using periodic structures," in Proceedings of the Loughborough Antennas and Propagation Conference (LAPC '08), pp. 93-96, Loughborough, UK, March 2008.

[23] E. Michailidis, C. Tsimenidis, and G. Chester, "Mutual coupling reduction in a linear two element patch array and its effect on theoretical MIMO capacity," in Proceedings of the Loughborough Antennas and Propagation Conference (LAPC '08), pp. 457-460, Loughborough, UK, March 2008.

[24] B. K. Lau, "Multiple antenna terminals," in MIMO: From Theory to Implementation, C. Oestges, A. Sibille, and A. Zanella, Eds., Elsevier, New York, NY, USA, 2010.
[25] C. G. Kakoyiannis, P. Gika, and P. Constantinou, "Small printed sinusoidal antennas: a simple design guide for smooth meander-line structures with augmented bandwidth," in Proceedings of the IEEE International Workshop on Antenna Technology (iWAT '09), Santa Monica, Calif, USA, March 2009.

[26] C. G. Kakoyiannis, P. Gika, and P. Constantinou, "Compact multi-element antennas of sinusoidal printed monopoles for sensors and portable devices," in Proceedings of the $3 r d$ European Conference on Antennas and Propagation (EuCAP '09), pp. 3423-3427, Berlin, Germany, March 2009.

[27] C. G. Kakoyiannis and P. Constantinou, "Coupling and correlation reduction in compact arrays for WSN nodes via pre-fractal defected ground plane," in Proceedings of the $3 \mathrm{rd}$ International Conference on Sensor Technologies and Applications (SENSORCOMM '09), pp. 175-180, Athens, Greece, June 2009.

[28] C. G. Kakoyiannis and P. Constantinou, "Reducing coupling in compact arrays for WSN nodes via pre-fractal defected ground structures," in Proceedings of the 39th European Microwave Conference (EuMC '09), pp. 846-849, Rome, Italy, SeptemberOctober 2009.

[29] C. G. Kakoyiannis, G. Stamatiou, and P. Constantinou, "Small square meander-line antennas with reduced ground plane size for multimedia WSN nodes," in Proceedings of the $3 \mathrm{rd}$ European Conference on Antennas and Propagation (EuCAP '09), pp. 2411-2415, Berlin, Germany, March 2009.

[30] C. G. Kakoyiannis and P. Constantinou, "Ground plane considerations for printed monopole antennas integrated into wireless sensor devices," in Proceedings of the International Wireless Communications and Mobile Computing Conference (IWCMC '08), pp. 738-743, Crete Island, Greece, August 2008.

[31] C. G. Kakoyiannis and P. Constantinou, "Co-design of antenna element and ground plane for printed monopoles embedded in wireless sensors," in Proceedings of the 2nd International Conference on Sensor Technologies and Applications (SENSORCOMM '08), pp. 413-418, Cap Esterel, France, August 2008.

[32] D. M. Pozar, "A relation between the active input impedance and the active element pattern of a phased array," IEEE Transactions on Antennas and Propagation, vol. 51, no. 9, pp. 2486-2489, 2003.

[33] S. Blanch, J. Romeu, and I. Corbella, "Exact representation of antenna system diversity performance from input parameter description," Electronics Letters, vol. 39, no. 9, pp. 705-707, 2003.

[34] J. Thaysen and K. B. Jakobsen, "Design considerations for low antenna correlation and mutual coupling reduction in multi antenna terminals," European Transactions on Telecommunications, vol. 18, no. 3, pp. 319-326, 2007.

[35] C.-S. Kim, J.-S. Park, D. Ahn, and J.-B. Lim, "A novel 1-D periodic defected ground structure for planar circuits," IEEE Microwave and Guided Wave Letters, vol. 10, no. 4, pp. 131133,2000

[36] D. Ahn, J.-S. Park, C.-S. Kim, J. Kim, Y. Qian, and T. Itoh, "A design of the low-pass filter using the novel microstrip defected ground structure," IEEE Transactions on Microwave Theory and Techniques, vol. 49, no. 1, pp. 86-93, 2001.

[37] J.-S. Park, "An equivalent circuit and modeling method for defected ground structure and its application to the design of microwave circuits," Microwave Journal, vol. 46, no. 11, pp. 2244, 2003.

[38] M.-S. Joung, J.-S. Park, and H.-S. Kim, "A novel modeling method for defected ground structure using adaptive frequency sampling and its application to microwave oscillator 
design," IEEE Transactions on Magnetics, vol. 41, no. 5, pp. 1656-1659, 2005.

[39] N. C. Karmakar, S. M. Roy, and I. Balbin, "Quasi-static modeling of defected ground structure," IEEE Transactions on Microwave Theory and Techniques, vol. 54, no. 5, pp. 2160$2168,2006$.

[40] Y. Chung, S.-S. Jeon, S. Kim, D. Ahn, J.-I. Choi, and T. Itoh, "Multifunctional microstrip transmission lines integrated with defected ground structure for RF front-end application," IEEE Transactions on Microwave Theory and Techniques, vol. 52, no. 5, pp. 1425-1432, 2004.

[41] C. G. Kakoyiannis, S. V. Troubouki, and P. Constantinou, "Comparison of efficient small antennas for wireless microsensors through simulation and experiment," in Proceedings of the 3rd International Symposium on Wireless Pervasive Computing (ISWPC '08), pp. 213-217, Santorini, Greece, May 2008.

[42] L. H. Weng, X. W. Shi, Y. C. Guo, C. X. W. Shi, and X. Q. Chen, "An overview on defected ground structure," Progress in Electromagnetics Research B, vol. 7, pp. 173-189, 2008.

[43] C. Mehlführer, S. Caban, and M. Rupp, "MIMO HSDPA throughput measurement results in an urban scenario," in Proceedings of the 70th IEEE Vehicular Technology Conference (VTC '09), Anchorage, Alaska, USA, September 2009.

[44] T. Weiland, M. Timm, and I. Munteanu, "A practical guide to 3-D simulation," IEEE Microwave Magazine, vol. 9, no. 6, pp. 62-75, 2008. 

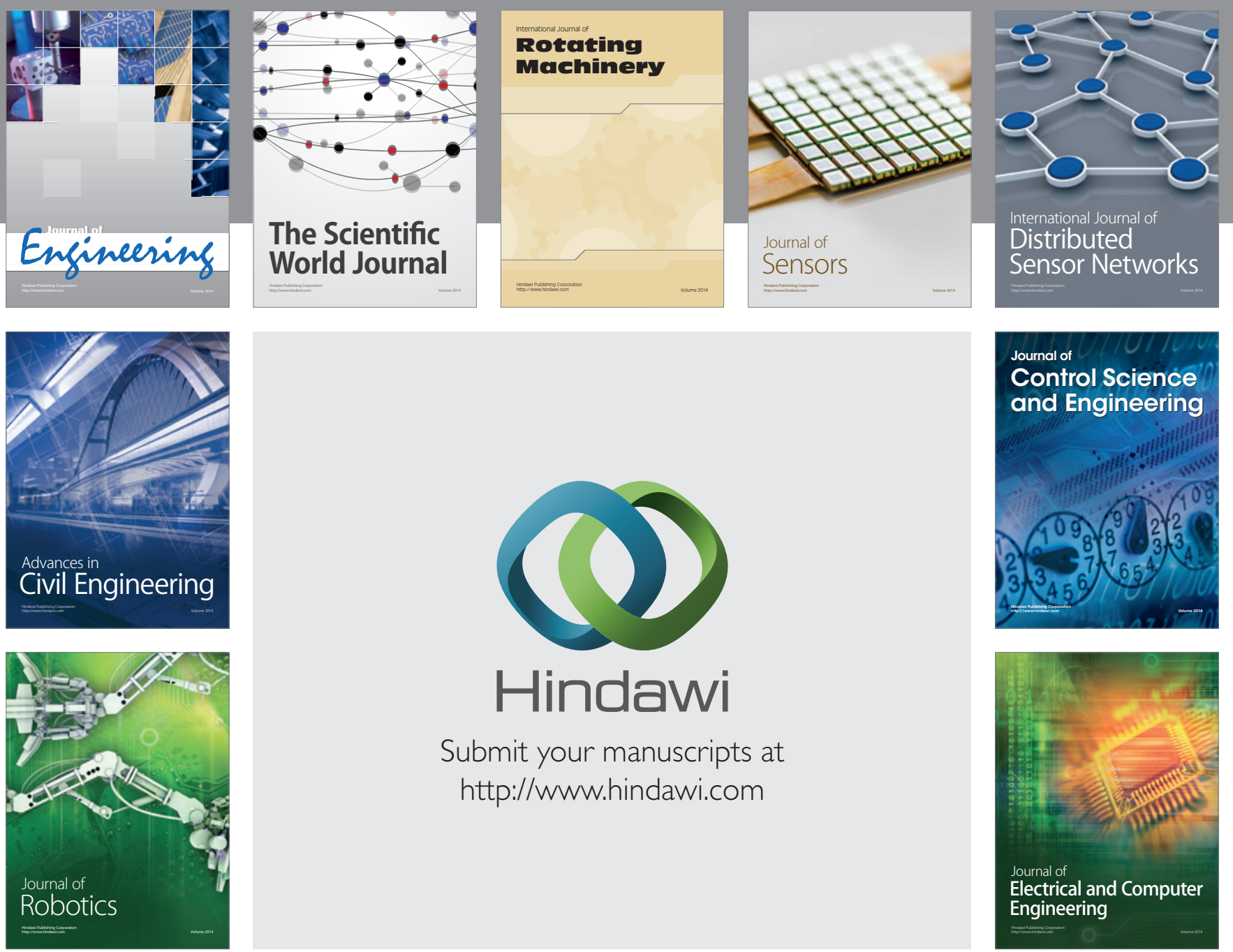

Submit your manuscripts at

http://www.hindawi.com
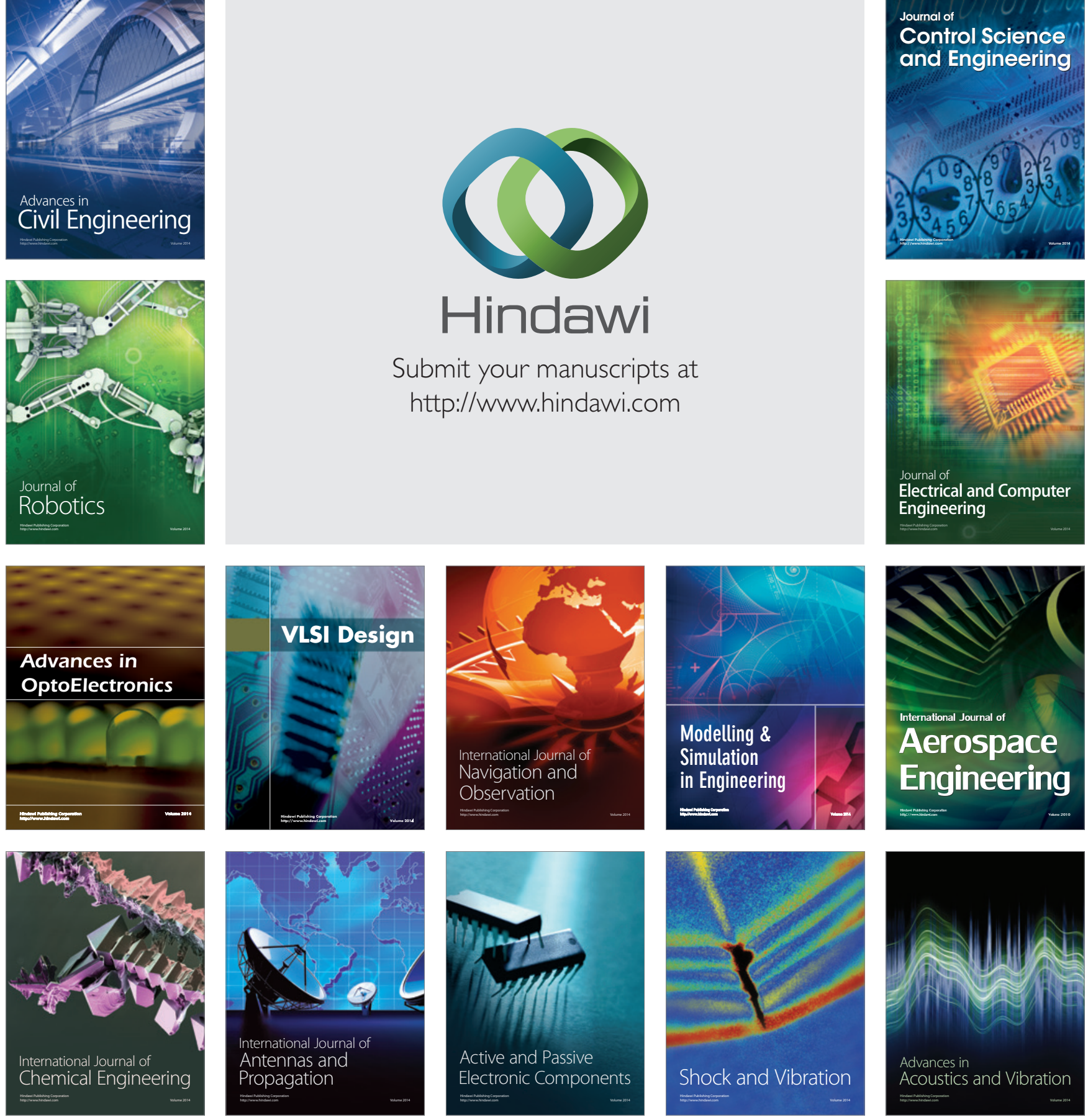